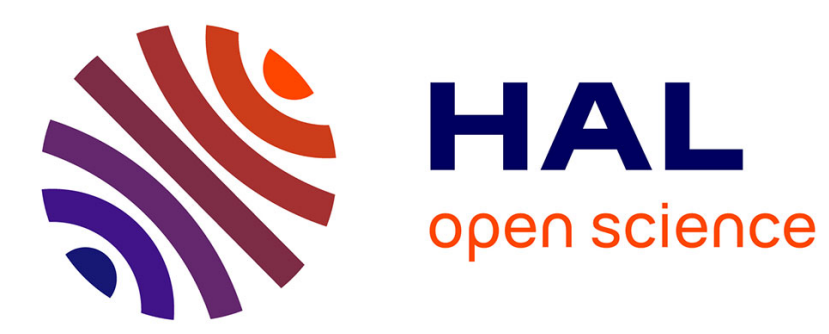

\title{
Advanced Modelling and Verification Techniques Applied to a Cluster File System
}

Charles Pecheur

\section{To cite this version:}

Charles Pecheur. Advanced Modelling and Verification Techniques Applied to a Cluster File System. RR-3416, INRIA. 1998. inria-00073273

\section{HAL Id: inria-00073273 \\ https://hal.inria.fr/inria-00073273}

Submitted on 24 May 2006

HAL is a multi-disciplinary open access archive for the deposit and dissemination of scientific research documents, whether they are published or not. The documents may come from teaching and research institutions in France or abroad, or from public or private research centers.
L'archive ouverte pluridisciplinaire HAL, est destinée au dépôt et à la diffusion de documents scientifiques de niveau recherche, publiés ou non, émanant des établissements d'enseignement et de recherche français ou étrangers, des laboratoires publics ou privés. 
INSTITUT NATIONAL DE RECHERCHE EN INFORMATIQUE ET EN AUTOMATIQUE

\section{Advanced Modelling and Verification Techniques Applied to a Cluster File System}

Charles Pecheur

$\mathbf{N}^{\circ} 3416$

May 1998

THÈME 1 



\title{
RIN RIA
}

\section{Advanced Modelling and Verification Techniques Applied to a Cluster File System}

\author{
Charles Pecheur* \\ Thème 1 - Réseaux et systèmes \\ Projet VASY \\ Rapport de recherche n3416 - May 1998 - 55 pages
}

\begin{abstract}
:
This report describes the application of elaborated formal modelling techniques and tools from the CADP toolset for LOTOS to the validation of CFS, a distributed file system. After a short overview of the LOTOS specification of CFS, we describe the techniques used for model generation and validation, and their application to CFS. Two original aspects are put forth: firstly, the model is generated in a compositional way, by putting together separately generated sub-components; secondly, the extensible, data-aware temporal logic checker XTL is used to express and validate properties of the system. In particular, an XTL extension providing richer diagnostics is presented. The full commented LOTOS specification is provided in appendix.
\end{abstract}

Key-words: Formal Method, Specification, Model Checking, Distributed File System, Compositional Generation, Temporal Logic, LOTOS, XTL.

(Résumé : tsvp)

Short version of this report in Charles Pecheur, "Advanced Modelling and Verification Techniques Applied to a Cluster File System", submitted for publication.

* Charles.Pecheur@inria.fr

Unité de recherche INRIA Rhône-Alpes

655, avenue de l'Europe, 38330 MONTBONNOT ST MARTIN (France)

Téléphone : 0476615200 - International: +33476615200

Télécopie : 0476615252 - International: +33476615252 


\section{Techniques avancées de modélisation et de vérification appliquées à un système de gestion de fichiers en grappe}

\section{Résumé :}

Ce rapport relate l'application de techniques et d'outils de modélisation formelle élaborés, appartenant à la boîte à outils CADP pour LOTOS, pour la validation du système de fichiers réparti CFS. Après un court aperçu de la spécification de CFS en LOTOS, nous décrivons les techniques utilisées pour générer et valider les modèles, et leur application à CFS. Deux aspects originaux sont mis en évidence : premièrement, le modèle est généré de manière compositionnelle, en assemblant des composants générés séparément; deuxièmement, le vérificateur de logique temporelle XTL, extensible et supportant les données, est utilisé pour exprimer et valider les propriétés du système. En particulier, on présente une extension de XTL fournissant des diagnostics enrichis. La spécification LOTOS complète et commentée est fournie en appendice.

Mots-clé : Méthode Formelle, Spécification, Vérification de Modèles, Système de Fichiers Réparti, Génération Compositionnelle, Logique Temporelle, LOTOS, XTL. 


\section{Introduction}

The benefits of formal methods for the design of complex distributed systems are now widely acknowledged. Many formalisms, algorithms and tools have been proposed for formally describing concurrent applications, expressing their properties and automating their verification. Two main approaches have been extensively studied: theorem proving and model checking. The latter, while applicable only to systems with a finite state space, offers the advantage of requiring much less participation from the user.

One should not conclude that model checking reduces to writing a specification and calling the checker, though. The well-known state space explosion is always lurking, and significant results only come out from the combination of large computing resources, sophisticated tools and skilled formal method experts.

This report illustrates the use of advanced techniques for the modelling and verification of CFS (Cluster File System) [Fas96], a distributed file system built on top of the ARIAS shared memory architecture [DHMdP96]. Two original aspects are put forth:

- the use of compositional model generation [FKM93], in order to produce a model that would have been impossible to generate in a single step, and

- the use of the extensible temporal logic checker XTL [Mat98] and the development of an $\mathrm{XTL}$ extension providing richer diagnostics.

The rest of this first section gives a survey of the LOTOS [ISO88] specification language and the CADP /Eucalyptus toolset [Gar96], which have been used in this project. Section 2 presents the CFS system along with its specification, Section 3 describes the compositional technique used to generate a model from this specification, and Section 4 discusses the verification task, including the development of an extension for the XTL checker.

\section{$1.1 \quad$ Overview of LOTOS}

Lotos is a standardized Formal Description Technique intended for the specification of communication protocols and distributed systems. Its design was motivated by the need for a language with a high abstraction level and a strong mathematical basis, which could be used for the description and analysis of complex systems. As a design choice, Lotos consists of two "orthogonal" sub-languages:

The data part is based on the well-known theory of algebraic abstract data types [Gut77], more specifically on the ACT ONE specification language [dMRV92]. Data types are defined using an equational formalism, which we will not present here. Indeed, most data types of our specification are defined in a higher level language, which is automatically translated into plain ACT ONE [Pec96].

The control part is based on a process algebra, combining the best features of CCS [Mil89] and CsP [Hoa85]. A concurrent system is described as a collection of processes interacting by rendez-vous. The behaviour of each process is built compositionally using 
an algebra of operators (choice, parallel composition,...) Behaviours can manipulate data values and exchange them through their interactions.

This report does not assume familiarity with LоTоs from the reader; the few forthcoming commented Lotos excerpts should be self-explanatory. Table 1 describes the main Lotos operators. Tutorials for Lotos are available, e.g. [BB88, Tur93].

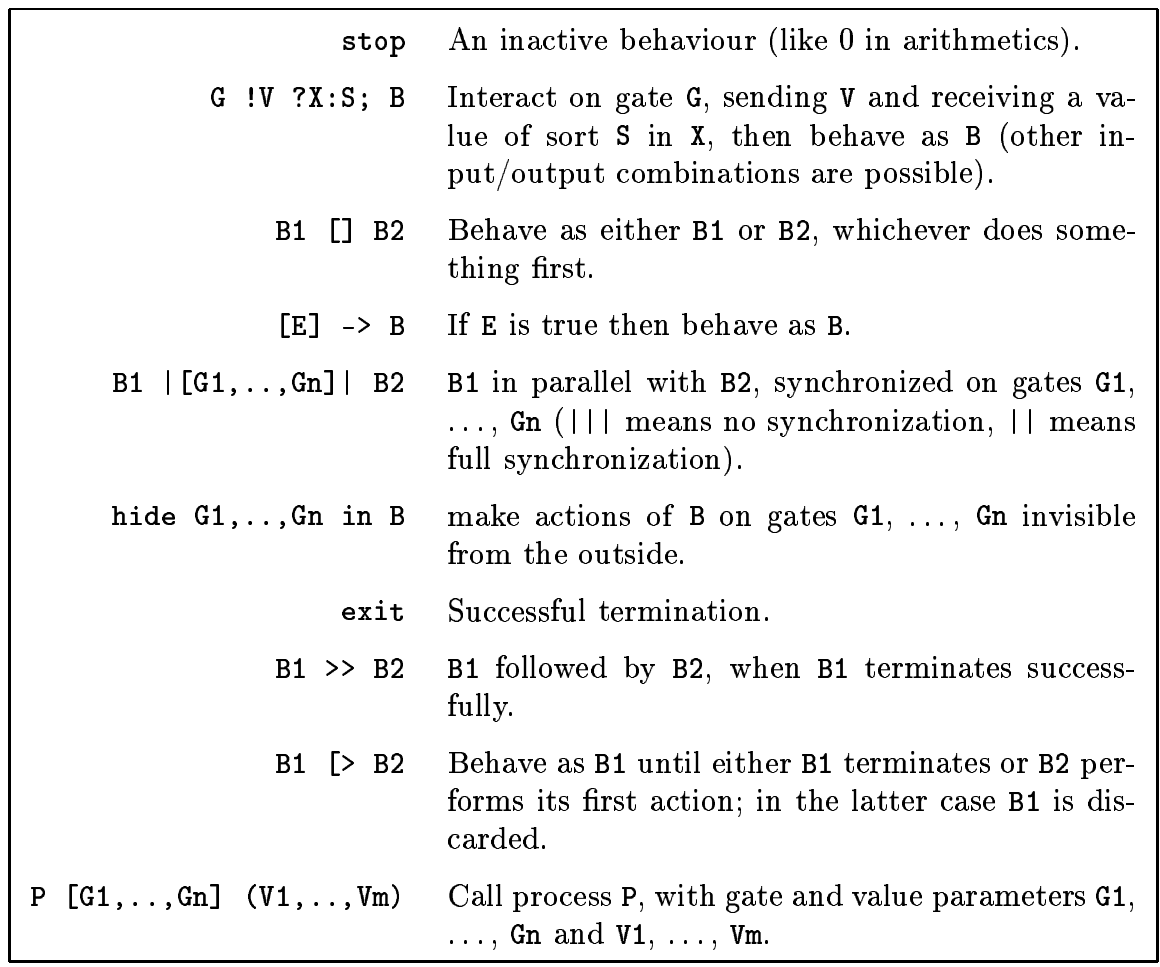

Table 1: Main LOTOS operators

The model (i.e. the meaning) of a LoTos specification is defined as the graph of all its possible actions (technically, this kind of graph is called a Labelled Transition System, or LTS, but we will keep the simpler words "model" and "graph" in this article). Models can be compared according to different equivalence and refinement criteria. In this study, we use observational equivalence [Mil89] for minimization, that is, we reduce models into minimal observationally equivalent ones.

Lотоs has been applied to many complex systems such as network services and protocols [ISO89, L94] but also cryptographic protocols [LBK ${ }^{+}$96] or hardware architectures $\left[\mathrm{CGM}^{+} 96\right]$. A number of tools have been developed for Lotos, covering user needs in such various areas as edition, simulation, compilation, test generation and formal verification. 


\subsection{The EUCALYPTUS/CADP Toolset}

All the work reported in this report has been done within the framework of the EUCALYPTus Lotos Toolset [Gar96], an X-Windows based, user-friendly interface federating several complementary Lotos tools from different sources. An important part of the EUCALYPTus toolset is CADP (CÆSAR/AldÉBaran Development Package) [FGK $+96, \mathrm{GJM}^{+}$97], a leading edge toolbox dedicated to the formal validation of distributed systems.

CADP offers an integrated set of functionalities ranging from interactive simulation to exhaustive, model-based verification methods, and includes sophisticated approaches to deal with large case-studies. In addition to LoTos, it also supports lower-level formalisms such as finite state machines and networks of communicating automata. In this case study, the following CADP tools were used:

- CæSAR [GS90] compiles the control part of a Lotos program into its transition graph. The data part is translated by the CÆSAR.ADT compiler [Gar89] into executable C code, which is used to compute the graph.

- Aldébaran [Fer89] is a verification tool for comparing or minimizing graphs with respect to any of several simulation and bisimulation relations.

- Open/Cesar [Gar98] is an open programming interface allowing to explore a graph in a controlled way. Several CAdP tools, such as XSimulator and Generator described below, are based on this technology. The CÆSAR compiler can produce OPEN/CÆSAR code allowing on-the-fly execution of a LoTOS specification. EXP.OPEN is another OPEN/CÆSAR code producer, giving access to a network of models of communicating processes.

- XSimulator is an interactive program for exploring the behaviour of a Lotos specification. It allows to walk through the alternative branches of the graph, using back and forth step-by-step execution.

- Generator performs an exhaustive exploration and generates the complete graph of a model. It thus plays a similar role as CÆSAR but can be applied to other OPEN/CÆSAR sources, such as networks of processes in combination with ExP.OpEN.

- XтL [Mat98] is a programmable temporal logic checker, based on a specialized functional programming language equipped with primitives for graph exploration. Definitions of several well-known temporal logics such as ACTL and the modal $\mu$-calculus are provided, and new ones can easily be added. Further details about XTL are given in Section 4 .

The Eucalyptus toolbox also contains the APERo data type pre-processor [Pec96]. This compiler provides convenient concise syntax extensions for declaring many common families of data structures such as records, enumerations, sets, lists, as well as general MLstyle constructor declarations. APERo translates these declarations into standard Lotos 
type definitions, equipped with all the usual associated operations (constructors, selectors, equality, etc.).

\section{Specification of CFS}

\subsection{Presentation of ARIAS and CFS}

ARIAS [DHMdP96] is a shared memory support system implemented as an extension of the AIX operating system. It provides a virtual memory among a set of machines, in such a way that applications share a unique address space. Rather than using a single coherence protocol that would be expensive and overly restrictive for most applications, ARIAS allows such protocols to be plugged into the system as specialization modules according to the needs of specific applications, resulting in better performance. The ARIAS memory space is composed of fixed size blocks (called zones in ARIAS) that are the smallest units of shared access.

The Cluster File System (CFS) [Fas96] is a distributed file system built on top of ARIAS, with the double purpose of validating the ARIAS system itself and experimenting with distributed applications that use shared files as a programming paradigm. The resulting structure is illustrated in Figure 1, where the shaded areas are not covered by the LoTos specification.

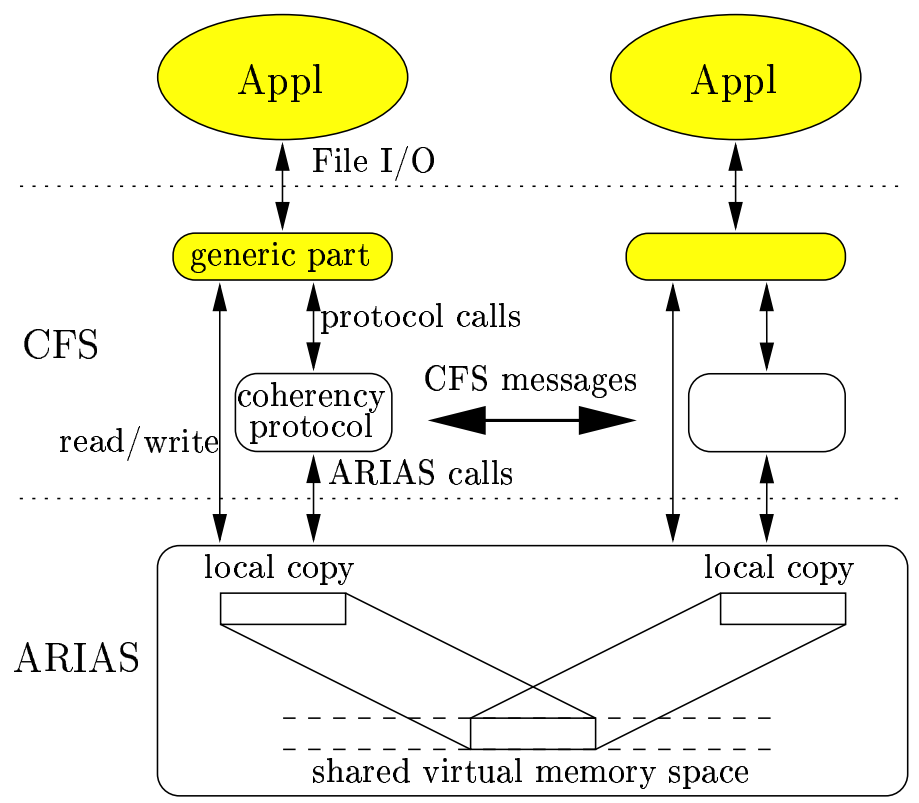

Figure 1: ARIAS and CFS 
Several file coherency protocols can co-exist, using different specialization modules. In practice, four coherency protocols have been implemented in CFS. Among them, the migratory protocol is designed to take full advantage of the ARIAS system and stands out after the multiple benchmarks described in [Fas96]. The specification and verification work presented here focuses on that protocol, which is referred to as the "CFs protocol" in the sequel. The CFS protocol relies on the notion of mastership, inherited from ARIAS: at any time, a master site owns the reference copy of the block data. Mastership can move between sites during the lifetime of the block; this accounts for much of the flexibility offered by ARIAS. Every CFs protocol message goes either from some slave to the master or vice-versa.

\subsection{Structure of the Specification}

To perform model-based verification, we need to generate a model of finite (and tractable) size. This puts constraints on the LoTos specification: for example, the number of parallel processes must be statically bounded, and choices over infinite ranges are forbidden. Furthermore, various parameters (data ranges, buffer sizes, etc.) are set to minimal values to keep the size of the model within reachable bounds.

The CFS protocol manages each block of memory independently, so our LoTOs specification focuses on the management of a single block (because of this, the block address field in all interactions never changes and is therefore omitted in the specification). Both the CFS protocol and the ARIAS service that is used by this protocol are specified. The size of the system is fixed to three sites: this is both an imposed maximum w.r.t. state space explosion and a requested minimum w.r.t. the coverage of possible scenarios in the system (some interesting situations do not occur with only two sites).

Most data types have been defined using the APERO syntax extensions. Besides reducing the size of data type declarations ( 82 vs. 433 lines of data type definitions), these notations are also much more readable, avoid the burden of equational definitions and hide the technical complications needed to allow the compilation of algebraic data type definitions. The complete specification (with APERO notations) is about 1000 lines long, and is provided in appendix at the end of this report.

The specification models the CFS protocol at two different levels of abstraction:

- the process CFS covers the control level, where we consider only the calls to CFS primitives for acquiring and releasing access to the CFS block;

- the process Complete adds complementary processes to CFS to compose the data level, where we also take into account access to and modification of the data in the block of memory itself.

The top-level structure of the resulting specification is shown on Figure 2.

The CFS protocol itself sits at the control level, and is specified for a given site as a process Site with four gates: cfsreq and cfsans support calls from the applications, as request/answer pairs of events, and send and rcv support emission and reception of CFS protocol messages through the underlying ARIAs system. At this level, the behaviour of 


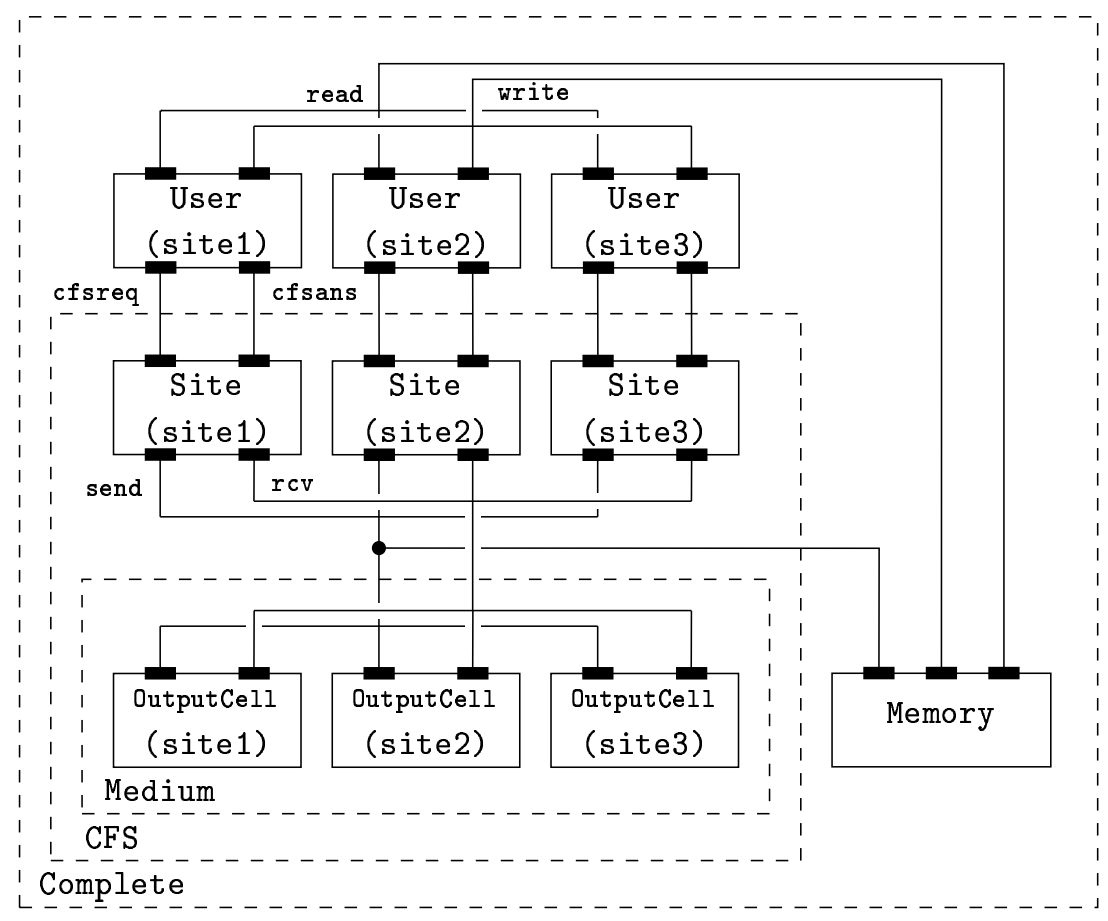

Figure 2: Structure of the Lotos specification of CFS

ARIAS is reduced to a communication facility, described in the process Medium. For modelling purposes, the buffering capacity is limited to one message per sending site: Medium is made of single-slot queues OutputCell put in parallel, one per site.

The data level part in process Memory holds the local copies of the block at each site and applies read and write events on them. It also observes the messages passing through the send gate and propagates master copies accordingly, i.e. following any readok or writeok message. The size of the model will depend on the cube of the range of possible block values (since there are three sites and thus three local copies): this range has therefore been reduced to only two values.

To complete the picture, a process User for each site enforces the correct use of CFS synchronization primitives: for example, write events are only allowed between beginwrite and endwrite CFS calls (this is an abstraction of the shaded upper layer of CFS on Figure 1).

Figure 3 gives an illustration of how these different parts work together. 


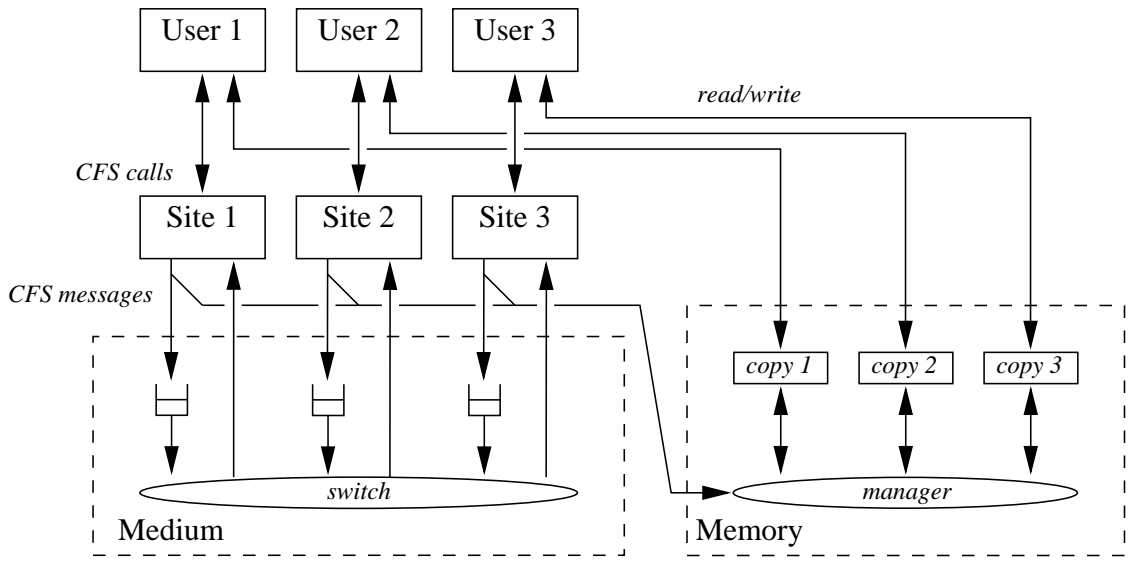

Figure 3: ARIAS service for CFS

\subsection{The CFS Protocol}

The source CFS definition [Fas96] describes the protocol as an input-output state automaton. The definition of process Site essentially captures that automaton, although several rounds of discussions with the designers were needed to reach the level of accuracy needed for formal specification. For illustration, Figure 4 shows the automaton corresponding to the specification: ! $m$ (resp. ? $m$ ) stands for emission (resp. reception) of $m$, CFs calls are in bold (vs. CFs messages), and $\{m\}$ denotes a repetition of $m$. Deadlocks detected in early models revealed several missing cases in the original description, drawn as dotted edges.

In the LOTOS specification of CFS, the state variables of the protocol become parameters of the Lотоs process. The state itself is also encoded as a parameter (this produces smaller models than defining different mutually recursive processes for each state). The body of Site is a non-deterministic choice between reception of CFS requests and messages. For example, the reception of a readok message is specified as:

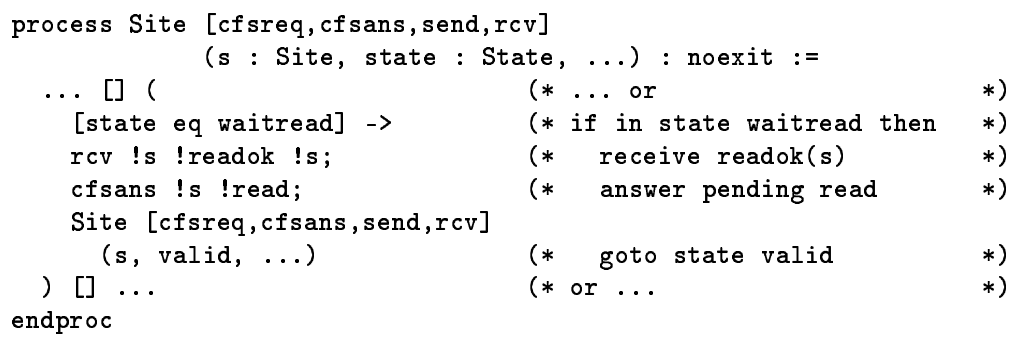




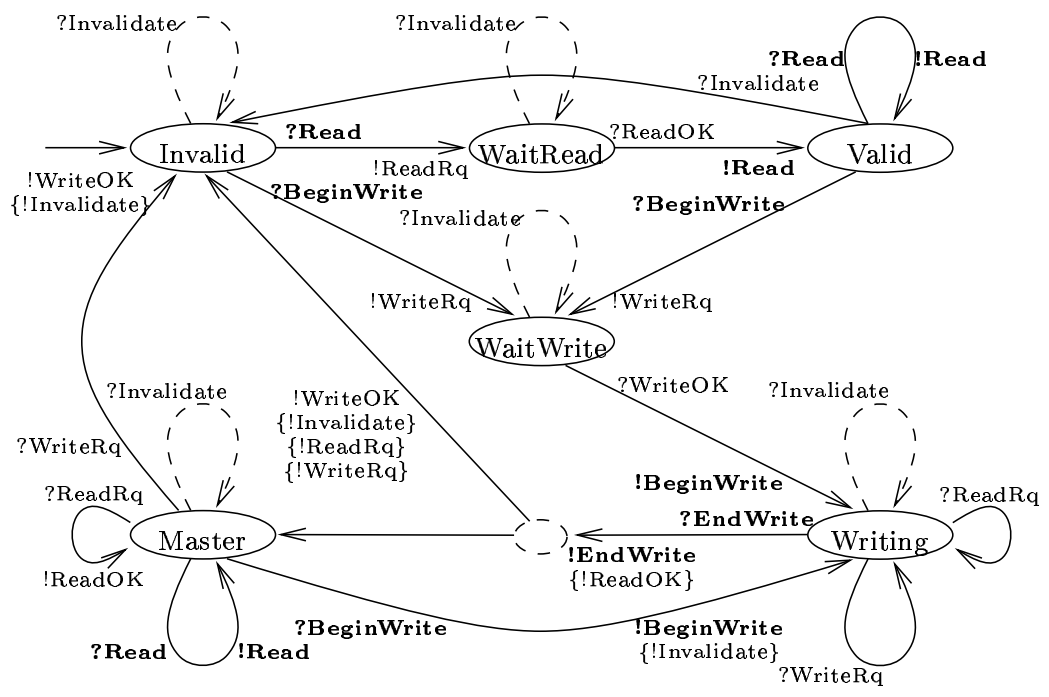

Figure 4: State of a block in the CFs protocol

\section{Model Generation}

The resulting LoTos specification of CFs has a finite model, but is too complex to be compiled in a monolithic way using available tools and computers. Indeed, early attempts on the control part alone produced a (essentially unusable) model with 2.7 million states and 9.2 million transitions. Instead of this, we used a divide-and-conquer approach, compiling sub-components of the system separately before combining them together, while minimizing each intermediate model before using it. This section explains this compositional technique and discusses its qualities and limits.

\subsection{Tools for Compositional Model Generation}

Compositional generation can be handled in CADP through the EXP.OPEN tool. EXP. Open takes as input a LoTos-like behaviour expression using only parallel and hiding operators, describing a network of communicating automata. It allows the exploration of the resulting model by any OPEN/CÆSAR tool. In particular, GENERATOR can be used to produce the explicit representation of that model. Concretely, this appears as a single program invocation, in which object code produced by EXP.OPEN is linked with library code for GENERATOR and executed. Using this technique, compositional generation is obtained through the following steps: 
1. generate the model for each component of the system using CÆSAR and minimize it (modulo observational equivalence) using AldÉBARAN,

2. combine some components using EXP.OpEn, produce the combined graph with GENERATOR and minimize it using AldÉBARAN,

3. repeat the previous step until obtaining the model of the whole system.

AldÉBARAN is also able to handle networks of communicating automata, merging the combination and minimization phases. However, usage has shown that using EXP.OPEN and Generator and then minimizing the resulting graph with AldÉBARAN is more efficient, in both terms of memory and time.

The delicate part of compositional generation, however, is to decide where to cut the whole system into separate components and in which order to combine them. Indeed, two interacting parts $P \| Q$ generally strongly constrain each other's behaviour, and generating $P$ or $Q$ separately can produce a much larger graph than $P \| Q$ itself, or even an infinite one, thus compromizing the approach.

To overcome this, a solution is to synchronize $P$ with an environment $E_{P}$, so that $P \| E_{P}$ produces a smaller model that can be substituted for $P$. This is sound provided that the substitution does not modify the global model. In turn, this is guaranteed if $E_{P}$ is a conservative approximation of the rest of the system as seen from $P$, i.e. if $E_{P}$ allows all executions that $P$ can go through as part of the whole system.

Let us mention that CADP provides a tool called Projector [KM97] that implements a closely related principle and is even able to control the validity of the environment $E_{P}$. However, Projector loops infinitely on some components of the CFs model and thus could not be used here.

\subsection{Generation of a Model of CFS}

From the specification depicted on Figure 2, a complete model of the management of a single CFS block has been generated compositionally, using environments to reduce the initial sizes of processes User and OutputCell. Besides models for Complete and its components, including CFS, The following models have also been generated:

Abstract $=$ Complete where only gates read and write remain visible. This gives a very abstract view in terms of values read and written in memory, while assuming (because of User processes) that CFS primitives are called appropriately.

Abstract2 $=$ two concurrent instances of Abstract, modelling read/write access to two different blocks. An "address" attribute is added to the events of each instance to distinguish them.

Table 2 gives the sizes of the different components and the generation and minimization times (in seconds, on a Sun Ultra-1 workstation).

RR n3416 
Table 2: Model generation statistics

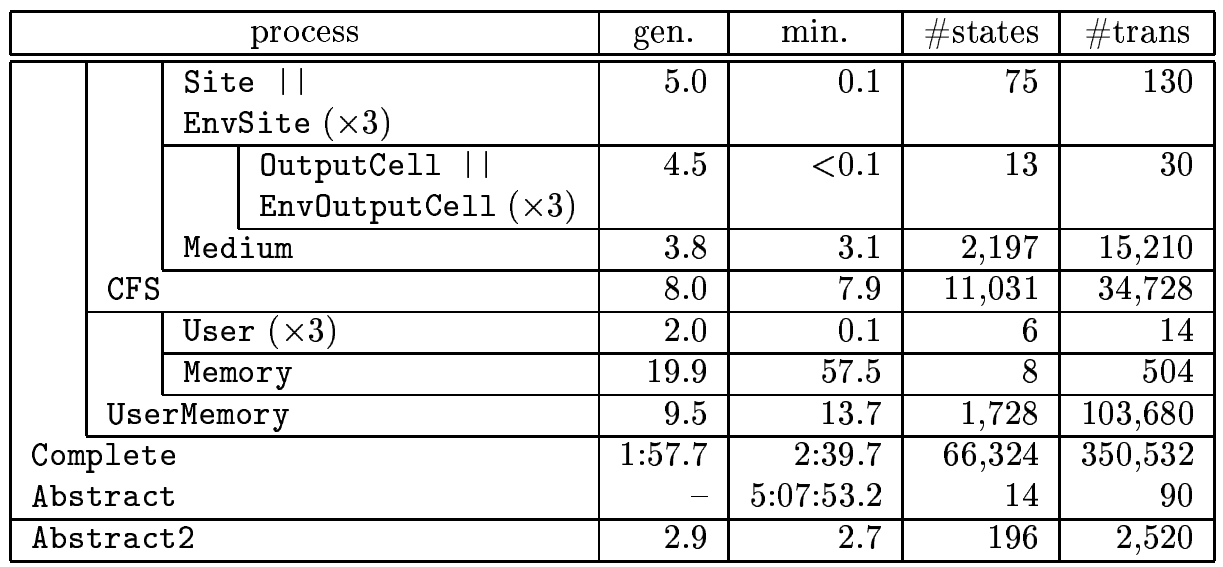

It is worth noting that our simplifications (three sites, single-slot communication channels, two block values) affect dimensions in the system itself only, not the behaviour of its environment (the correlation imposed by User processes is part of the definition of CFs). In this sense, we obtain a general model of the system, in contrast with some of our other previous experiments [Pec97] in which only restricted and finite scenarios have been modelled.

The model obtained for process Abstract is particularly interesting: it is a highly symmetrical graph with only 14 states, shown in Figure 5 (labels are abbreviated for clarity: R (resp. W) $! s ! v$ stands for read (resp. write) value $v$ from site $s$ ). In states 0 and 11 , all local copies have the same value. The three outgoing edges correspond to any of the sites changing that value, and the following internal transitions show the propagation of the new value to other sites.

From a technical point of view, the two copies of Abstract combined to produce Abstract2 were obtained by relabelling the Abstract model using the UNIX sed utility. This solution is much cheaper than generating each copy separately from the source LOTOS process.

A fully non-synchronized composition as in Abstract2 is hit headlong by exponential inflation, however: for $n$ independent components, the number of states is $S_{n}=S_{1}{ }^{n}$ and the number of transitions is $T_{n}=n \cdot T_{1} \cdot S_{1}{ }^{n-1}$. According to this, combining two concurrent instances of Complete would go far beyond available computing resources. 


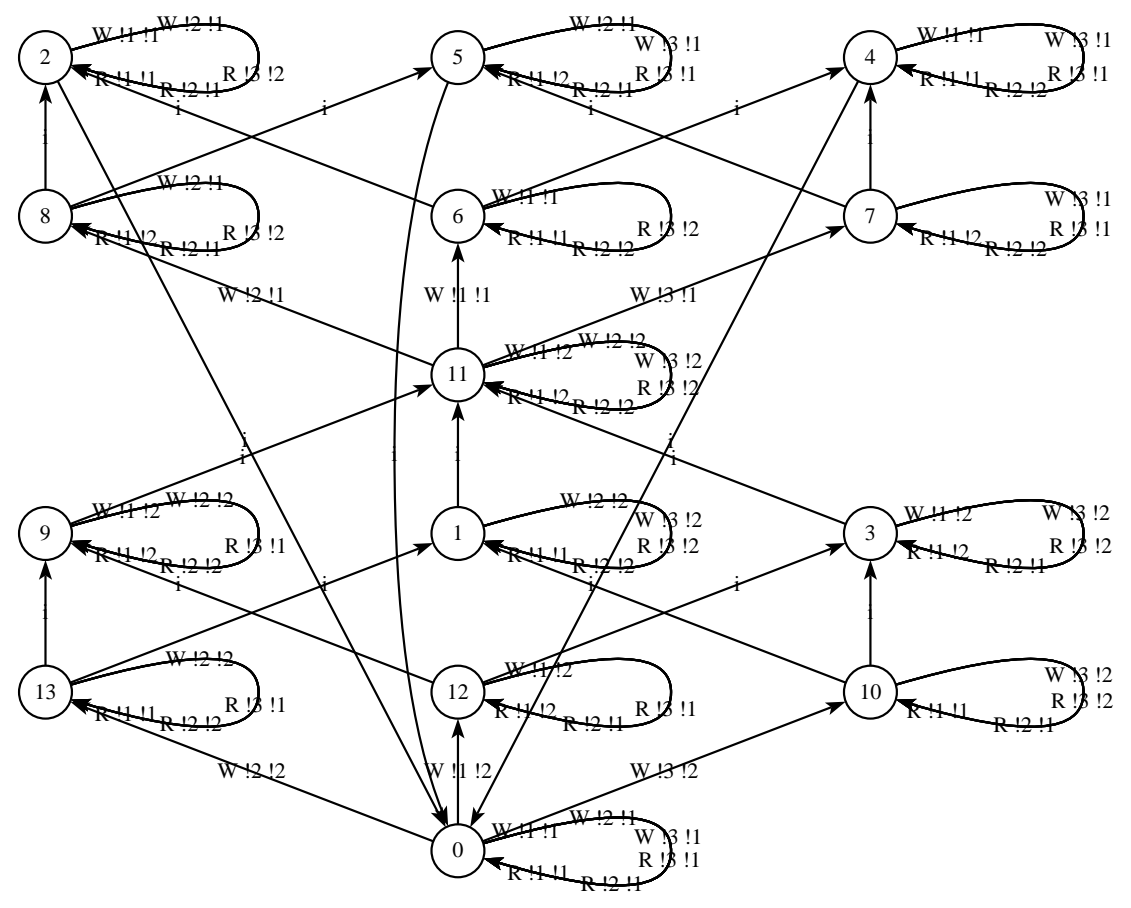

Figure 5: Model of the Abstract process

\section{Verification}

The properties of the CFS protocol have been expressed and evaluated as temporal logic formulas, using the $\mathrm{XTL}$ tool. This section gives an overview of $\mathrm{XTL}_{\mathrm{TL}}$, describes how temporal logic operators have been re-defined to obtain more detailed evaluation results, and discusses the verification of the CFS specification using XTL.

\subsection{Overview of XTL}

Though primarily intended for evaluation of temporal logic formulas, $\mathrm{XTL}$ is in full generality a compiler for a functional language applied to a labelled transition system. The XTL language is equipped with data types for states, transitions and labels, and sets thereof, and functions for manipulating them (e.g. initial state, incoming and outgoing transitions of a state, source and target states of a transition). For example, the following XTL expression computes the set of all non-deterministic states:

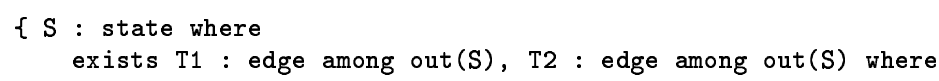




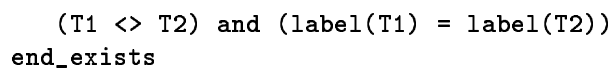

It can also do pattern matching on the labels of transitions and thus access the individual attributes of structured LoTos events. The following example searches for some transition on $\mathrm{G}$ with integer attribute larger than 10 :

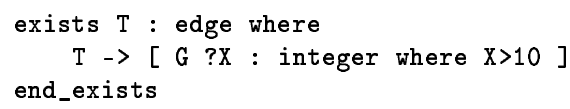

Results are reported using a side-effect print function. Temporal operators are defined as functions and/or macros using these primitives; definitions for standard logics (e.g. HML, ACTL, modal $m u$-calculus) are provided as XTL libraries. We have used (a fragment of) the ACTL logic [NV90], which has four primitive temporal operators (besides usual boolean connectors):

$$
F::=\mathrm{EX}_{A} F\left|\mathrm{AX}_{A} F\right| \mathrm{E}\left[F_{A} \mathrm{U} F\right] \mid \mathrm{A}\left[F_{A} \mathrm{U} F\right]
$$

The following table gives their meaning, their XTL syntax, and introduces a few other derived operators used in this report:

\begin{tabular}{|c|c|c|c|}
\hline XTL syntax & math syntax & definition & meaning \\
\hline 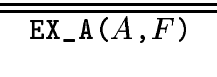 & $\overline{\mathrm{EX}_{A} F}$ & (primitive) & $\begin{array}{l}\text { Some path does an } A \text { step that } \\
\text { reaches } F \text {. }\end{array}$ \\
\hline $\mathrm{AX} \_\mathrm{A}(A, F)$ & $\mathrm{AX}_{A} F$ & (primitive) & $\begin{array}{l}\text { All paths do an } A \text { step that reaches } \\
F \text {. }\end{array}$ \\
\hline EU_A $(F, A, G)$ & $\mathrm{E}\left[F_{A} \cup G\right]$ & (primitive) & $\begin{array}{l}\text { Some path stays in } F \text { through } A \\
\text { steps until it reaches } G \text {. }\end{array}$ \\
\hline $\mathrm{AU} \_\mathrm{A}(F, A, G)$ & $\mathrm{A}\left[\begin{array}{ll}F & \cup \\
\mathrm{U}\end{array}\right]$ & (primitive) & $\begin{array}{l}\text { All paths stay in } F \text { through } A \text { steps } \\
\text { until they reach } G \text {. }\end{array}$ \\
\hline $\operatorname{Dia}(A, F)$ & $\langle A\rangle F$ & $\mathrm{EX}_{A} F$ & Some $A$ step reaches $F$. \\
\hline $\operatorname{Box}(A, F)$ & {$[A] F$} & $\neg \mathrm{EX}_{A} \neg F$ & All $A$ steps reach $F$. \\
\hline $\mathrm{EF}_{-} \mathrm{A}(A, F)$ & $\mathrm{EF}_{A} F$ & $\mathrm{E}\left[\mathrm{tt}_{A} \cup F\right]$ & Some $A$ path reaches $F$. \\
\hline $\mathrm{EF}(F)$ & $\mathrm{EF} F$ & $\mathrm{EF}_{\mathrm{tt}} F$ & Some path reaches $F$. \\
\hline $\mathrm{AG} \_\mathrm{A}(A, F)$ & $\mathrm{AG}_{A} F$ & $\neg \mathrm{E}\left[\mathrm{tt}_{A} \mathrm{U} \neg F\right]$ & All $A$ paths stay in $F$. \\
\hline $\mathrm{AG}(F)$ & $\mathrm{AGF}$ & $\mathrm{AG}_{\mathrm{tt}} F$ & All paths stay in $F$ \\
\hline
\end{tabular}

\subsection{Generating diagnostics in XTL}

The standard libraries provided with XTL evaluate temporal operators by computing their denotational semantics, i.e. a temporal formula produces the set of states that satisfy it. For example, the $\mathrm{EX}_{A}$ operator, whose semantics is

$$
\llbracket \mathrm{EX}_{A} F \rrbracket=\left\{s \mid \exists s \stackrel{a}{\longrightarrow} s^{\prime} . a \in A \wedge s^{\prime} \in \llbracket F \rrbracket\right\}
$$

is defined in the standard XTL library as 


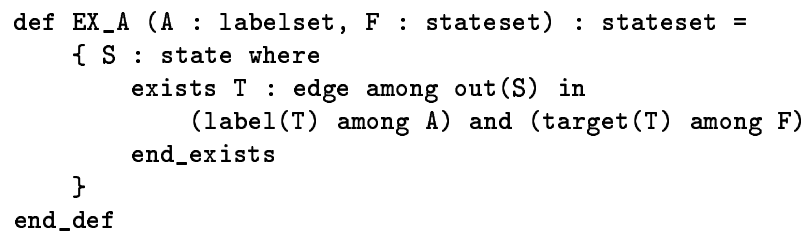

This approach gives a linear complexity w.r.t. the size of the formula, but provides no justification of why the computed states satisfy the formula. For example, when some state $s$ satisfies $\operatorname{EX}_{A} F$, we would like to exhibit a transition $s \stackrel{a}{\longrightarrow} s^{\prime}$ where $a$ is in $A$ and $s^{\prime}$ satisfies $F$. More generally, we seek a more sophisticated evaluation method that produces explanations [Ras91]: the evaluation of a temporal formula $F$ on a state $s$

- evaluates whether $F$ holds on $s$, and

- prints out a trace from $s$ that confirms the result whenever possible.

Apparently this turns formulas into predicates over states, and thus temporal operators into functions over predicates, which would require a higher-order language. Nevertheless, a similar effect can be achieved thanks to the availability of macros in XTL. We turn formulas into open boolean expressions with a free variable CURRENT containing the current state, and operators into macros. For example, $\mathrm{EX}_{A}$ becomes

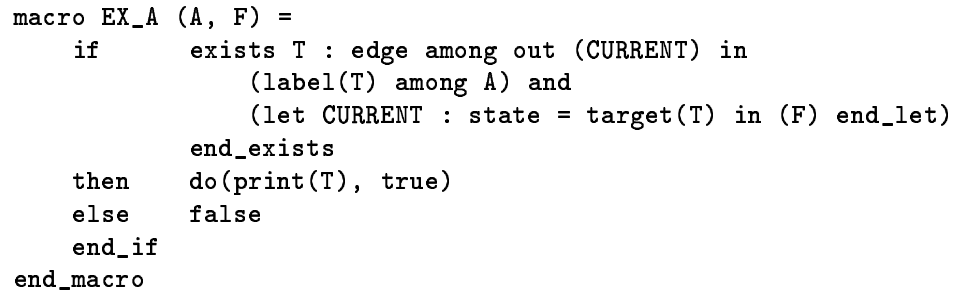

Assuming CURRENT contains some state $s$, T ranges over transitions $s \stackrel{a}{\longrightarrow} s^{\prime}$ such that $a$ is in A and $s^{\prime}$ satisfies $\mathrm{F}$. The let construct binds (a fresh incarnation of) CURRENT to $s^{\prime}$ ) before evaluating $\mathrm{F}$. The use of macros is essential: a function would evaluate $\mathrm{F}$ in the calling context instead, losing the opportunity to re-bind CURRENT. do $(a, x)$ is a macro call that performs action $a$ then returns $x$.

The other basic macro implements $\mathrm{E}\left[F_{A} \mathrm{U} G\right]$. The XTL code uses general iteration operators and will not be detailed here. In summary, the macro EU_A (F, A, G) performs a breadth-first search from CURRENT for a state that satisfies $G$ through edges that match $F$ and $A$, and stores the search tree. When a successful state is reached, it follows and prints a path through the search tree from that state back to the start state.

Since the search is breadth first, a shortest path is reported. This is a very useful property in practice, because facilitates the interpretation of the diagnostic: shortest paths contain the minimal sequence of events leading to the obtained result, whereas arbitrary 
paths may go through events that are irrelevent to the result being diagnosed. Combining several breadth-first searches does not necessarily result in the shortest overall trace, though.

All other operators can be defined in terms of (a slight generalization of) these two macros. In particular, since we work on finite models, infinite traces always end in circuits, which can be found using the following macro:

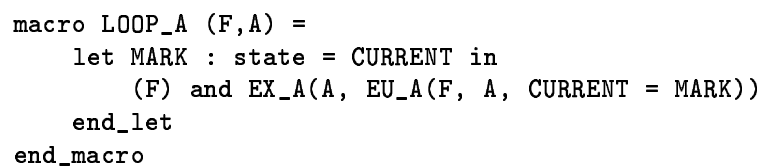

The body of LOOP_A keeps the start state in MARK, then searches for a path of at least one $A$ step that stays in $F$ and comes back to MARK.

Some evaluations of temporal formulas have no useful diagnostic trace: for example, if EX $_{A} F$ does not hold in $s$, it means that all traces fail to reach $F$ through an $A$ step, so all outgoing edges from $s$ should belong to the diagnostic. Such diagnostics generate huge output with little information; we do not attempt to produce them. In particular, ACTL operators come in pairs, performing dual quantification over traces: $E$ operators (i.e. EX and $\mathrm{E}\left[\mathrm{U}_{-}\right]$) search for the existence of one path satisfying certain criteria. Accordingly, the implementation of these operators will report that path as a diagnostic when the result is positive, but remain silent on negative results. Conversely, A operators will provide a diagnostic only on negative results. Nested E (or A) operators will produce consecutive pieces of a larger path. Conversely, an A nested inside an E should and will remain silent in all cases. A general discussion this issue can be found in [Ras91].

All diagnostic traces are produced "on the fly", that is, as soon as the XTL operator obtains the corresponding result. Because of this, the trace has to be printed backwards, since more deeply nested operators, which produce further parts of the trace, necessarily obtain their result first. Doing otherwise would require to store the trace and process it afterhand, which is currently not possible: XTL only supports sets (vs. lists) of edges, which are not adequate in to store traces (which may contain loops in all generality). This also restricts diagnostics to a single trace: for example, producing two diagnostic traces for conjunctive formulas $F \wedge G$ (one for $F$ and one for $G$ ) would require to store the first one until a second one is obtained.

The generation of diagnostics adds a cost, in both terms of memory and time: since all operators are macros, formulas expand into big XTL expressions, which can stretch the XTL compiler to its limits. The linear complexity is also lost: $n$ nested EU_A will produce $n$ nested breadth-first searches, with an exponential worst-case complexity $O\left(k^{n}\right)$, where $k$ is the size of the model. This is the price to pay to obtain diagnostic traces within the current XTL implementation. Things can be improved by pre-computing sub-formulas that will never be traced, although this requires a finer analysis from the user.

On the other hand, because everything gets expanded into a single expression, bound variables can be used in nested sub-formulas. This allows to capture and refer to intermediate states in the exploration, as illustrated in the LOOP_A macro above, and even more interes- 
tingly to propagate attribute values, allowing to express things such as "any message sent is eventually received". The verification of CFs below shows the usefulness of this possibility.

\subsection{Properties of CFS}

Besides generic properties such as absence of deadlocks and non-determinism, our verification work focuses on the read/write coherency properties of the CFS protocol. These properties are expressed at the data level, in terms of events on gates read and write.

We proceed in two steps. First the formulas are evaluated on the Abstract model, where only the concerned gates are visible. The evaluation is fast since this model is very small. However the diagnostic traces are not informative because all the inner workings of the protocol have been abstracted. The interesting formulas are then evaluated again, this time on the Complete model. This takes longer but provides fully detailed diagnostic traces.

Since there is no CFs call for ending a read session, it was expected that the read/write coherency is rather loose. This coherency was not formally expressed at the start of this study, though, so the work consisted as much in determining the expected properties as in verifying them.

The following nine properties have been expressed and evaluated (the outcome of the evaluation is shown in parentheses):

1. Global Liveness: there is no global deadlock (holds).

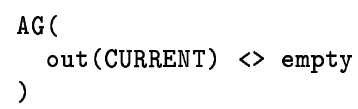

2. Determinism: no state has non-deterministic transitions (fails).

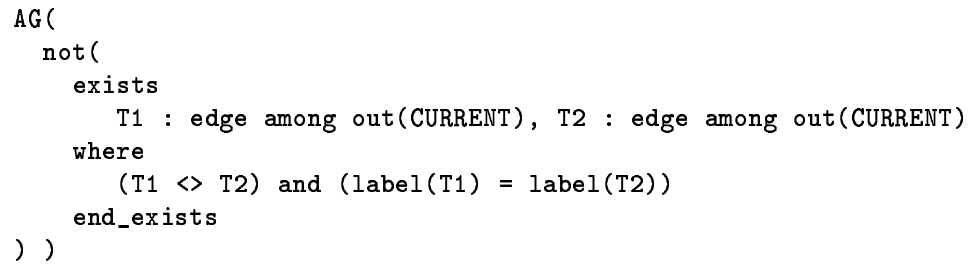

3. Local liveness: at any time, all sites can eventually read and write (holds).

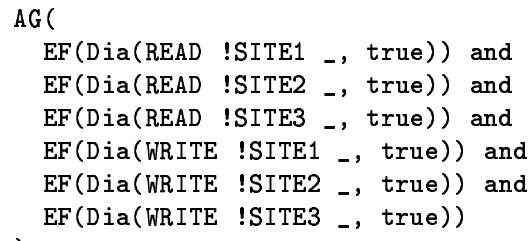


4. Atomic coherency: if no write occurs inbetween,

4a. two different sites always read the same value (fails).

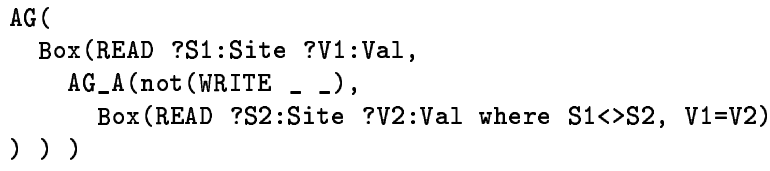

4b. a single site always reads the same value (fails).

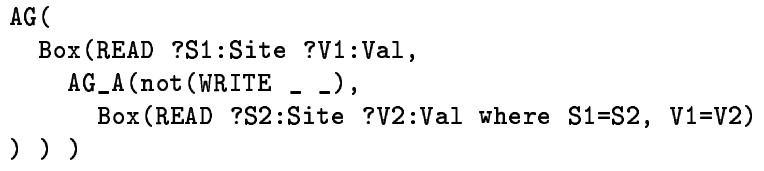

4c. if one site writes a value, another site will always read that value afterwards (fails).

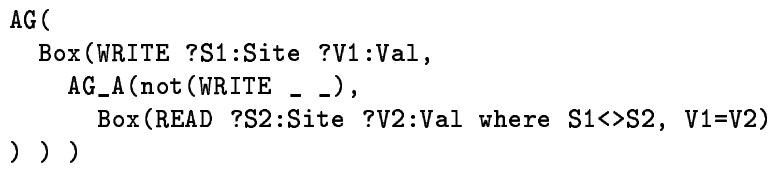

4d. if one site writes a value, the same site will always read that value afterwards (holds).

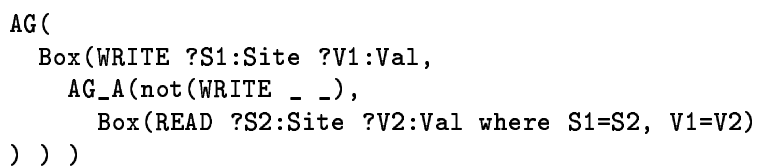

5. Propagation of values: assuming a fair execution, if one site writes a value and no one writes inbetween, all sites will eventually read that value afterwards (holds).

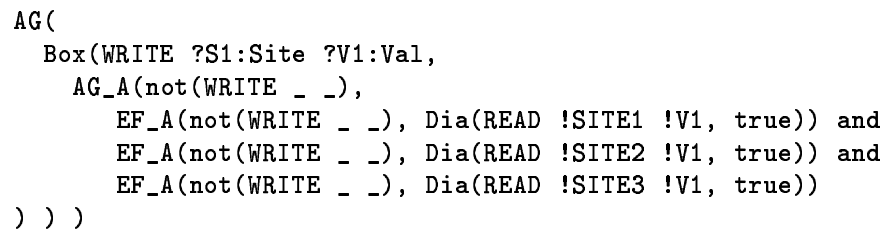

According to [QS83], we express the fairness assumption using the $\mathrm{AG}_{A} \mathrm{EF}_{A} F$ combination, which means: it is always possible to eventually reach $F$ while staying on $A$ paths, or yet, all fair executions of $A$ paths will eventually reach $F$. Without the fairness assumption, we would have written $\mathrm{AF}_{A} F$ instead $\left(=A\left[\mathrm{tt}_{A} \mathrm{U} F\right]\right)$. 
6. Sequential consistency: if one site writes a value, no one writes inbetween, and another site reads that value, it cannot read another value afterwards (holds).

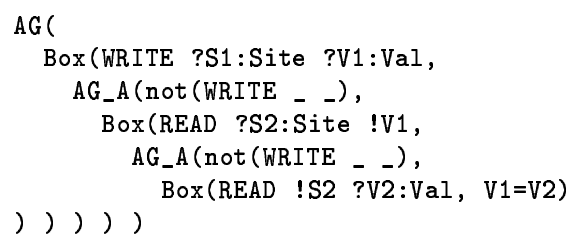

Compilation and evaluation of all nine formulas on the Abstract model takes 93 seconds of CPU time (on a Sun Ultra-1). As expected, evaluation on the Complete model takes much longer: 273 seconds and 52490 seconds (more than 14 hours) for properties 2 and $4 \mathrm{~b}$ to report diagnostic traces of length 32 and 21 , respectively. The trace produced for formula $4 \mathrm{~b}$ follows, as an illustration. The transitions $s \stackrel{a}{\longrightarrow} s^{\prime}$ are displayed as triples $\left(s, a, s^{\prime}\right)$, where $s$ and $s^{\prime}$ are given as internal state numbers.

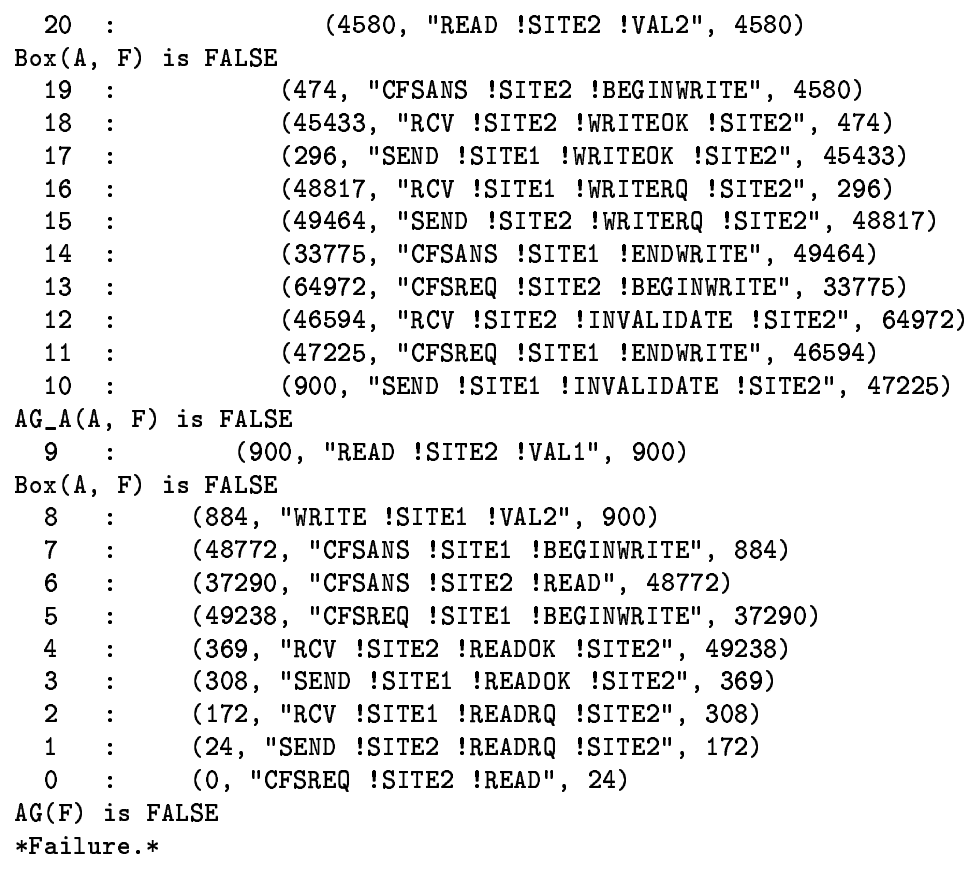

We use the possibility to bind and use attribute values to express properties 4 a to $4 \mathrm{~d}$, 5 and 6 in their full generality, each as a single formula. For example, in property $4 a$, the variables S1, V1, S2 and V2 are used to capture and compare the sites and values of two consecutive READ events. Without this possibility, we would have had to repeat the formula for all possible combinations of values of these variables.

Let us now comment some evaluation results: 
- Non-determinism is not a failure of the protocol but rather a consequence of the abstraction level of its LоTоs specification: diagnostic traces for property 2 show that the non-deterministic transitions correspond to possible reception of the same message from two different sources, a perfectly legal situation in the protocol.

- Failure of properties $4 \mathrm{a}, 4 \mathrm{~b}$ and $4 \mathrm{c}$ is not surprising, since this kind of atomic coherency is quite strong and the protocol was known to have loose read synchronization mechanisms.

- Property 6 is more characteristic of typical distributed memory systems. It is related to the notion of sequential consistency [Mos93], which requires that events in the system are seen in the same order on all sites, though the time scales can be stretched or shifted from one site to another. In essence, property 6 captures the fact that values are read in the order in which they are written. This is even stronger than sequential consistency, which would allow two unrelated writes on different sites to be seen (at all sites) in the opposite order than that in which they really occurred.

If several memory blocks are considered, however, sequential coherency does no longer hold. This is illustrated on model Abstract2 using the following property:

7. If one site writes values in two blocks in some order (and no other write occurs inbetween), another site cannot read the written value in the second block then fail to read the written value in the first block afterwards.

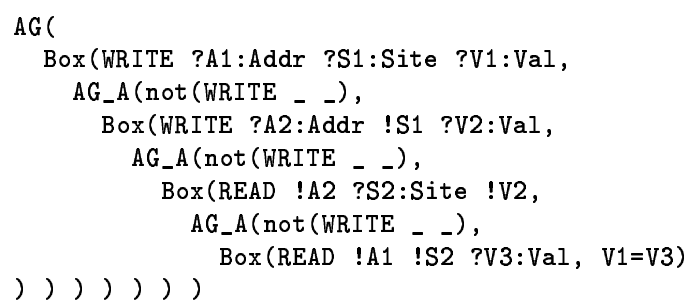

Property 7 is implied by sequential consistency and is not satisfied by Abstract2: intuitively, writes to different blocks can propagate at different speeds. CFS is thus sequentially consistent at the block level but not at the file level.

\section{Conclusions}

Although the LOTOS specification of CFS has a modest size, it produces excessively large models if processed in a straightforward manner. The modelling and verification work presented here illustrates how sophisticated tools can be used to achieve significant results in such complex cases. 
Strictly speaking, the restrictions and simplifications needed to obtain a model of tractable size restrict the generality of the results we obtain. In this sense, model checking can be considered as a (very powerful) debugger, that is, a tool to find problems rather than prove their absence. In practice though, is is reasonable to expect most properties to be stable w.r.t the size of the system.

Beyond the validation of the CFs protocol, this case study has also demonstrated the practical applicability and usefulness of two technologies for dealing with complex specifications and supported by the CADP toolset:

- Using AldéBARAn and Open/CÆsar technology to generate and compose models of different components of the system, we have been able to build up a model for a complete system that would have been impossible to produce in a single LоTos compilation.

- CFs properties have been expressed and evaluated using the XTL temporal logic checker, with two important advantages. Firstly, XTL supports data, so that properties about values exchanged in the system have been conveniently expressed. Secondly, the XTL language is extensible, so that we have been able to define new temporal operators that produce execution traces to illustrate their results.

[Mat98] provides all the technical basis for the next version of XTL which will, hopefully, allow properties to be expressed even more conveniently and evaluated even more efficiently.

\section{Acknowledgements}

This study has been carried in close collaboration with both ARIAS/CFS developers and CADP tool designers. In particular, we would like to mention Thierry Jacquin and Daniel Hagimont on the former side, Hubert Garavel and Radu Mateescu on the latter. Many thanks too to some of them for their judicious comments on this text. Norman Ramsey's NoweB literate programming system has been used to write the Lotos specification. AIX is a trademark of IBM Corporation. 


\section{References}

[BB88] Tommaso Bolognesi and Ed Brinksma. Introduction to the ISO Specification Language LOTOS. Computer Networks and ISDN Systems, 14(1):25-29, January 1988.

[CGM ${ }^{+}$96] Ghassan Chehaibar, Hubert Garavel, Laurent Mounier, Nadia Tawbi, and Ferruccio Zulian. Specification and Verification of the PowerScale Bus Arbitration Protocol: An Industrial Experiment with LOTOS. In Reinhard Gotzhein and Jan Bredereke, editors, Proceedings of the Joint International Conference on Formal Description Techniques for Distributed Systems and Communication Protocols, and Protocol Specification, Testing, and Verification FORTE/PSTV'96 (Kaiserslautern, Germany), pages 435-450. IFIP, Chapman \& Hall, October 1996. Full version available as INRIA Research Report RR2958 .

[DHMdP96] Pascal Dechamboux, Daniel Hagimont, Jacques Mossière, and Xavier Rousset de Pina. The Arias Distributed Shared Memory: an Overview. In 23rd Intl Winter School on Current Trends in Theory and Practice of Informatics, volume 1175 of Lecture Notes in Computer Science, 1996.

[dMRV92] Jan de Meer, Rudolf Roth, and Son Vuong. Introduction to Algebraic Specifications Based on the Language ACT ONE. Computer Networks and ISDN Systems, 23(5):363-392, 1992.

[Fas96] Jean-Philippe Fassino. Utilisation d'une mémoire virtuelle répartie pour le support d'un système de fichiers réparti. DEA, Université Joseph Fourier, Grenoble, June 1996.

[Fer89] Jean-Claude Fernandez. ALDEBARAN: A Tool for Verification of Communicating Processes. Rapport SPECTRE C14, Laboratoire de Génie Informatique — Institut IMAG, Grenoble, September 1989.

[FGK ${ }^{+96}$ Jean-Claude Fernandez, Hubert Garavel, Alain Kerbrat, Radu Mateescu, Laurent Mounier, and Mihaela Sighireanu. CADP (CÆSAR/ALDEBARAN Development Package): A Protocol Validation and Verification Toolbox. In Rajeev Alur and Thomas A. Henzinger, editors, Proceedings of the 8th Conference on Computer-Aided Verification (New Brunswick, New Jersey, USA), volume 1102 of Lecture Notes in Computer Science, pages 437-440. Springer Verlag, August 1996.

[FKM93] Jean-Claude Fernandez, Alain Kerbrat, and Laurent Mounier. Symbolic Equivalence Checking. In C. Courcoubetis, editor, Proceedings of the 5th Workshop on Computer-Aided Verification (Heraklion, Greece), volume 697 of Lecture Notes in Computer Science. Springer Verlag, June 1993. 
[Gar89] Hubert Garavel. Compilation of LOTOS Abstract Data Types. In Son T. Vuong, editor, Proceedings of the 2nd International Conference on Formal Description Techniques FORTE'89 (Vancouver B.C., Canada), pages 147-162. North-Holland, December 1989.

[Gar96] Hubert Garavel. An Overview of the Eucalyptus Toolbox. In Z. Brezočnik and T. Kapus, editors, Proceedings of the COST 247 International Workshop on Applied Formal Methods in System Design (Maribor, Slovenia), pages 76-88. University of Maribor, Slovenia, June 1996.

[Gar98] Hubert Garavel. OPEN/CÆSAR: An Open Software Architecture for Verification, Simulation, and Testing. In Bernhard Steffen, editor, Proceedings of the First International Conference on Tools and Algorithms for the Construction and Analysis of Systems TACAS'98, Lecture Notes in Computer Science, Berlin, March 1998. Springer Verlag. Full version available as INRIA Research Report RR-3352.

[GJM+97] Hubert Garavel, Mark Jorgensen, Radu Mateescu, Charles Pecheur, Mihaela Sighireanu, and Bruno Vivien. CADP'97 - Status, Applications and Perspectives. In Ignac Lovrek, editor, Proceedings of the 2nd COST 247 International Workshop on Applied Formal Methods in System Design (Zagreb, Croatia), June 1997.

[GS90] Hubert Garavel and Joseph Sifakis. Compilation and Verification of LOTOS Specifications. In L. Logrippo, R. L. Probert, and H. Ural, editors, Proceedings of the 10th International Symposium on Protocol Specification, Testing and Verification (Ottawa, Canada), pages 379-394. IFIP, North-Holland, June 1990.

[Gut77] J. Guttag. Abstract Data Types and the Development of Data Structures. Communications of the ACM, 20(6):396-404, June 1977.

[Hoa85] C. A. R. Hoare. Communicating Sequential Processes. Prentice-Hall, 1985.

[ISO88] ISO/IEC. LOTOS - A Formal Description Technique Based on the Temporal Ordering of Observational Behaviour. International Standard 8807, International Organization for Standardization - Information Processing Systems Open Systems Interconnection, Genève, September 1988.

[ISO89] ISO/IEC. LOTOS Description of the Session Protocol. Technical Report 9572, International Organization for Standardization - Open Systems Interconnection, Genève, 1989.

[Jac] Thierry Jacquin. Le protocole de cohérence mémoire de CFS. unpublished notes. 
[KM97] Jean-Pierre Krimm and Laurent Mounier. Compositional State Space Generation from Lotos Programs. In Ed Brinksma, editor, Proceedings of TACAS'g7 Tools and Algorithms for the Construction and Analysis of Systems (University of Twente, Enschede, The Netherlands), volume 1217 of Lecture Notes in Computer Science, Berlin, April 1997. Springer Verlag. Extended version with proofs available as Research Report VERIMAG RR97-01.

[L9́4] Luc Léonard. The LOTOS Specification of the Enhanced Transport Service. In The OSI95 Transport Service with Multimedia Support, pages 239-244 and 398-515. Springer Verlag, 1994.

[LBK $\left.{ }^{+} 96\right]$ Guy Leduc, Olivier Bonaventure, Eckhart Koerner, Luc Léonard, Charles Pecheur, and Didier Zanetti. Specification and verification of a TTP protocol for the conditional access to services. In Proceedings of 12th J. Cartier Workshop, Formal Methods and their Applications: Telecommunications, VLSI and Real-Time Computerized Control System, Montreal, Canada, October 1996.

[Mat98] Radu Mateescu. Vérification des propriétés temporelles des programmes parallèles. Thèse de Doctorat, Institut National Polytechnique de Grenoble, April 1998.

[Mil89] Robin Milner. Communication and Concurrency. Prentice-Hall, 1989.

[Mos93] David Mosberger. Memory Consistency Models. Operating Systems Review, 27(1):18-26, 1993.

[NV90] R. De Nicola and F. W. Vaandrager. Action versus State based Logics for Transition Systems. In Proceedings Ecole de Printemps on Semantics of Concurrency, volume 469 of Lecture Notes in Computer Science, pages 407-419. Springer Verlag, 1990.

[Pec96] Charles Pecheur. Improving the Specification of Data Types in Lotos. Doctorate thesis, University of Liège, November 1996. Collection of Publications of the Faculty of Applied Sciences, Nr 171.

[Pec97] Charles Pecheur. Specification and Verification of the CO4 Distributed Knowledge System Using LOTOS. In Michael Lowry and Yves Ledru, editors, Proceedings of the 12th IEEE International Conference on Automated Software Engineering ASE-97 (Incline Village, Nevada, USA), November 1997. Extended version available as INRIA Research Report RR-3259.

[QS83] Jean-Pierre Queille and Joseph Sifakis. Fairness and Related Properties in Transition Systems - A Temporal Logic to Deal with Fairness. Acta Informatica, 19:195-220, 1983.

[Ras91] Anne Rasse. Error diagnosis in finite state systems. In Proceedings of $C A V^{\prime} 91$, number 575 in Lecture Notes in Computer Science. Springer Verlag, 1991.

INRIA 
[Tur93] Kenneth J. Turner, editor. Using Formal Description Techniques - An Introduction to ESTELLE, LOTOS, and SDL. John Wiley, 1993.

RR n3416 


\section{Specification of the CFS coherency protocol in LOTOS}

\section{A Introduction}

This document contains the LoTOS specification of the CFS coherency protocol, presented in a litterate programming style. The specification covers access to a single block of a CFS file (a zone in CFs terminology), and describes both the CFs coherency protocol (see process Site) and the real transfer and access to file data (see process Memory).

Notational Convention The full Lotos code is provided, in the form of labelled chunks like the following sample ${ }^{1}$ :

(* ... some LOTOS text here ...*)

A chunk may contain references to other chunks, to be interpreted as textual inclusion:

$(* \ldots *)$

$\langle$ sample 26a $\rangle$

(* ...**)

The Lotos language is officially defined by the ISO standard 8807 [ISO88]. Tutorials can be found in [BB88, Tur93].

Model Generation This specification is intended for model-checking using the CADP validation tools [Gar96]. This has some consequences in the way it is written:

- To limit state space explosion, data types are kept as small as possible. In particular, small sets of constants are often used to model potentially large data domains.

- The behaviour part has a bounded synchronization structure (no recursion over parallelism), and the number of concurrent processes is kept to a minimum.

- Equations are written assuming sequential evaluation (i.e. the first applicable equation is applied). This often allows a drastic reduction of the number of equations, but relies on the particular evaluation strategy used by CADP. It is not to be interpreted according to the standard algebraic semantics of LoTOs.

\footnotetext{
${ }^{1}$ This is produced automatically using N. Ramsey's Noweb literate programming system.
} 
Data Type Syntax Extensions The APERo syntax extensions [Pec96] are used to shorten and clarify the definitions of data types. These notations are not standard Lotos; a translator is used to expand them into plain LоTos data type definitions (taking into account the requirements of $\mathrm{CADP}$ ).

\section{B Version History}

Version 1 First version, based on the automaton found in [Fas96], p. 52, plus [Jac]. Describes the synchronization part of different sites for one block (actual memory transfer is not covered). Different control states are modelled as different LоTоs processes.

Version 2 To tackle state space explosion, the different processes are merged in a single one, with the control state represented explicitly as a data variable.

Version 3 Add modelling of block contents. Since the latest revisions of version 2, model generation is handled compositionally, so we take less care into reducing the number of variables in processes. CÆSAR's inefficiency in state representation is eliminated in subsequent minimizations.

Version 4 Drastic housecleaning: all unused processes and definitions removed. Intended for final distribution.

\section{Data Types}

\section{C.1 Base Domains}

Booleans are used throughout.

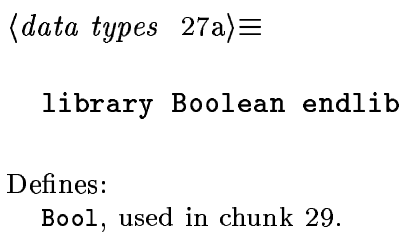

Each site is identified by an identifier of sort Site. This sort is defined as an enumerated type and is iterated upon in model generations; it should be kept as small as possible. This specification is bounded to three different sites. 
$27 \mathrm{~b}$

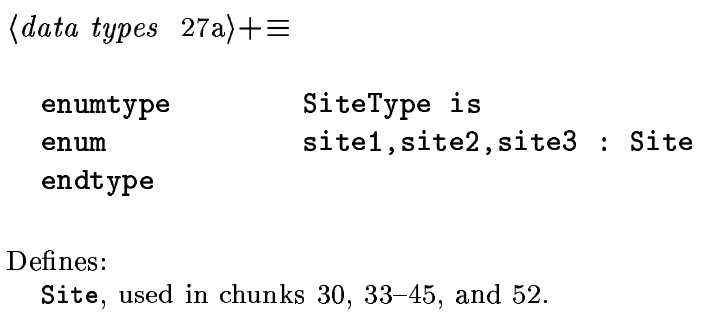

Val is the sort of block content. This sort is iterated upon and thus is kept as small as possible, i.e. two different values.

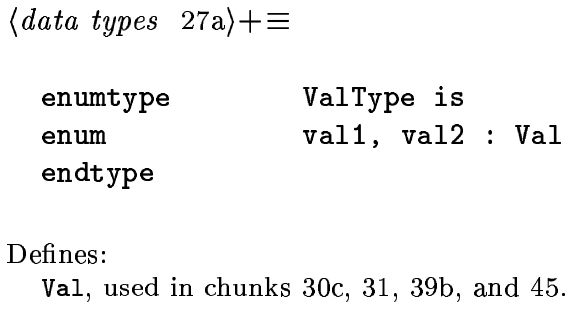

\section{C.2 Interaction Primitives}

CfsCall describes the CFs primitives offered to applications.

$28 \mathrm{~b}$

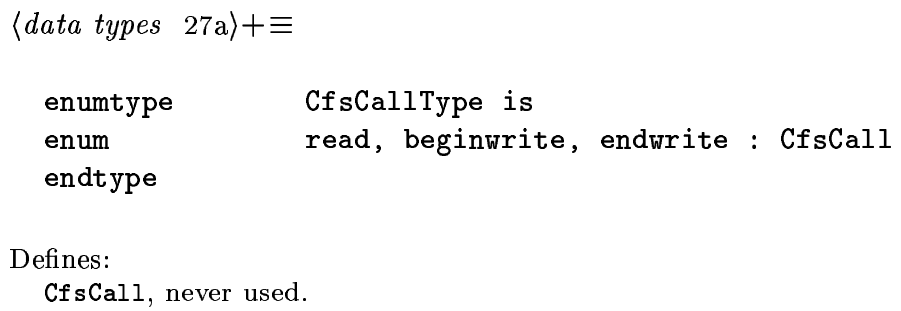

State is used in monitoring interactions, to observe the internal state of the different sites. The last four are transient states where internal information is processed; no message or request can be received in those states. 
- master The site is master, no one is writing.

- writing The site is master and in a writing session.

- invalid The site has no valid copy.

- valid The site owns a valid copy.

- waitread The site is waiting for a valid copy.

- waitwrite The site is waiting for mastership.

- flushrqs The site is master and is flushing pending requests (transient).

- forwardrqs The site has no valid copy and is forwarding pending requests to the current master (transient).

- invalwriting The site is invalidating remote copies before writing (transient).

- invalinvalid The site is invalidating remote copies while giving up mastership (transient).

$\langle$ data types $27 \mathrm{a}\rangle+\equiv$

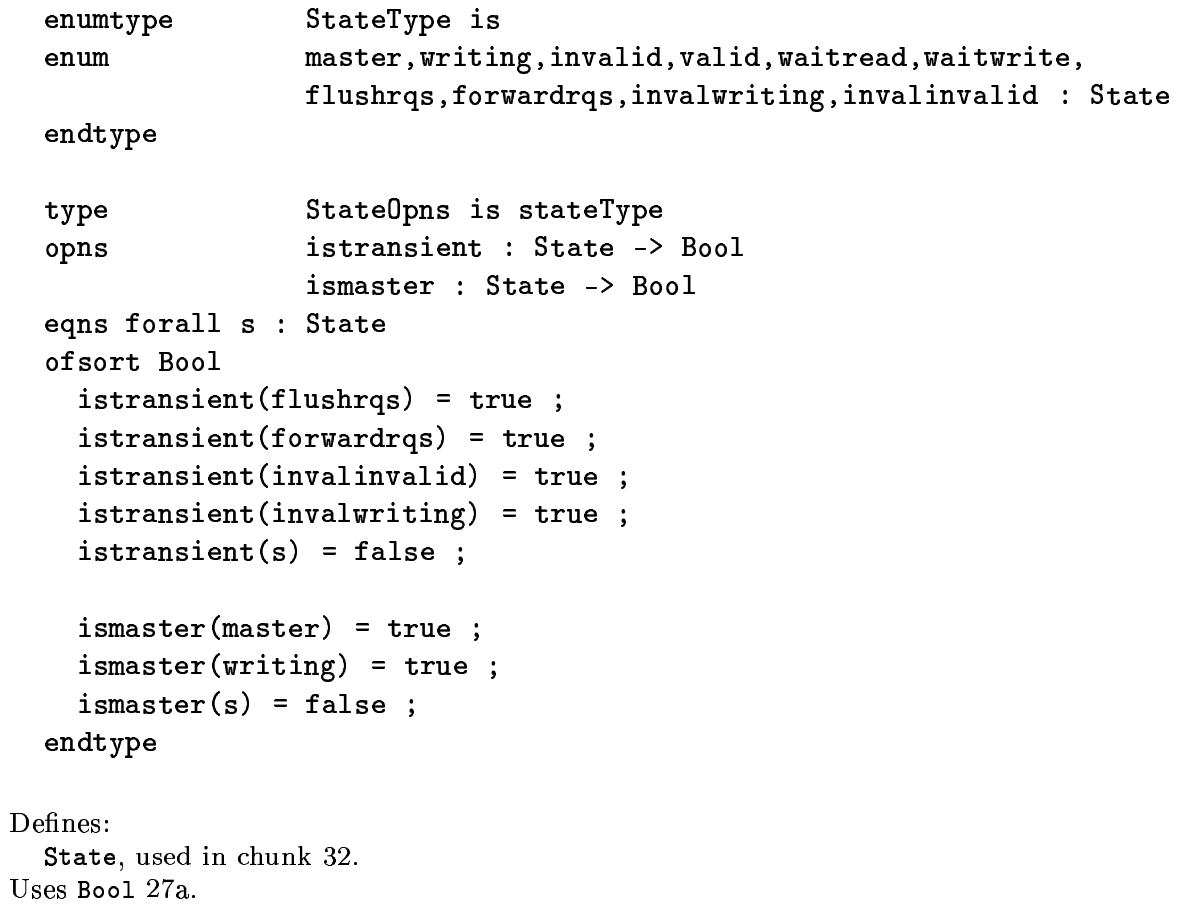

RR n3416 


\section{C.3 State Variables}

SiteSet defines sets of site identifiers, used by a block master to remember all remote copy requesters and holders.

$30 \mathrm{a}$

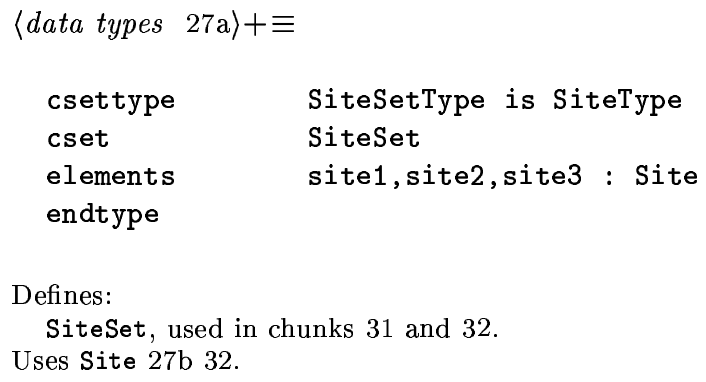

PktList defines a list of (Site, Message) pairs. It is used by the underlying communication channel to store transitting messages. the Site is the remote (i.e. non-master) site; it can be either the source or the destination of the message, depending on the message type.

$30 \mathrm{~b}$

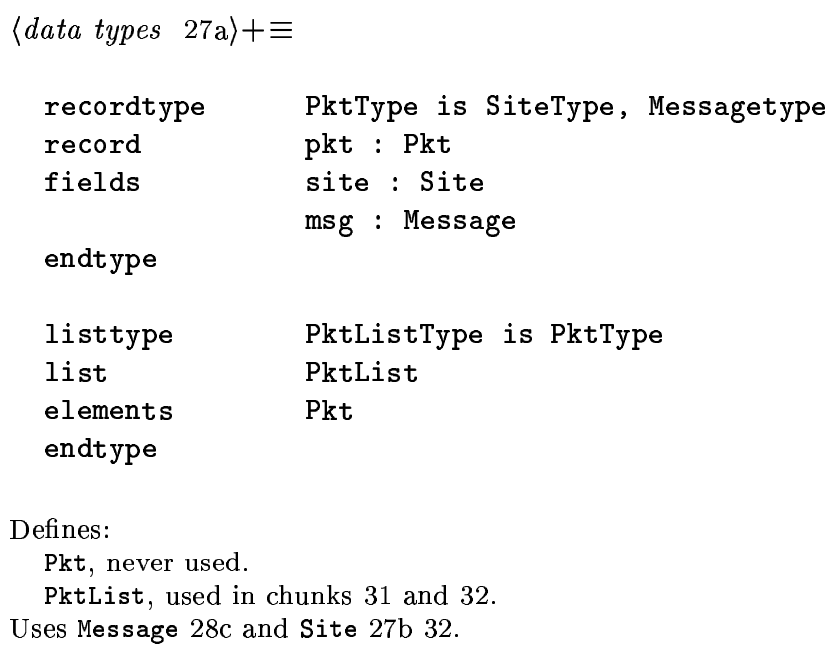

ValArray is an array of Val indexed on Site, used in process Memory to store the different copies of a block for each site.

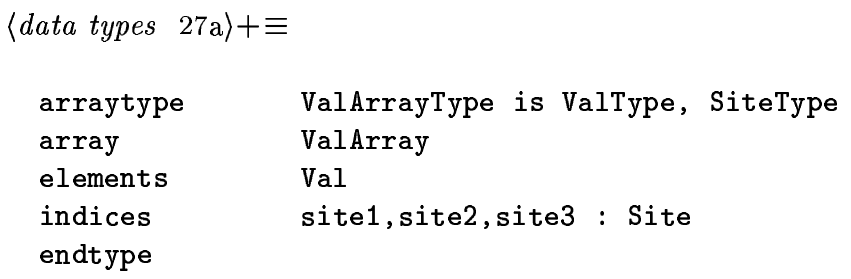


Defines:

ValArray, used in chunks 31 and $39 \mathrm{~b}$.

Uses Site 27b 32 and Val 28a.

Some complementary constants for convenience.

31

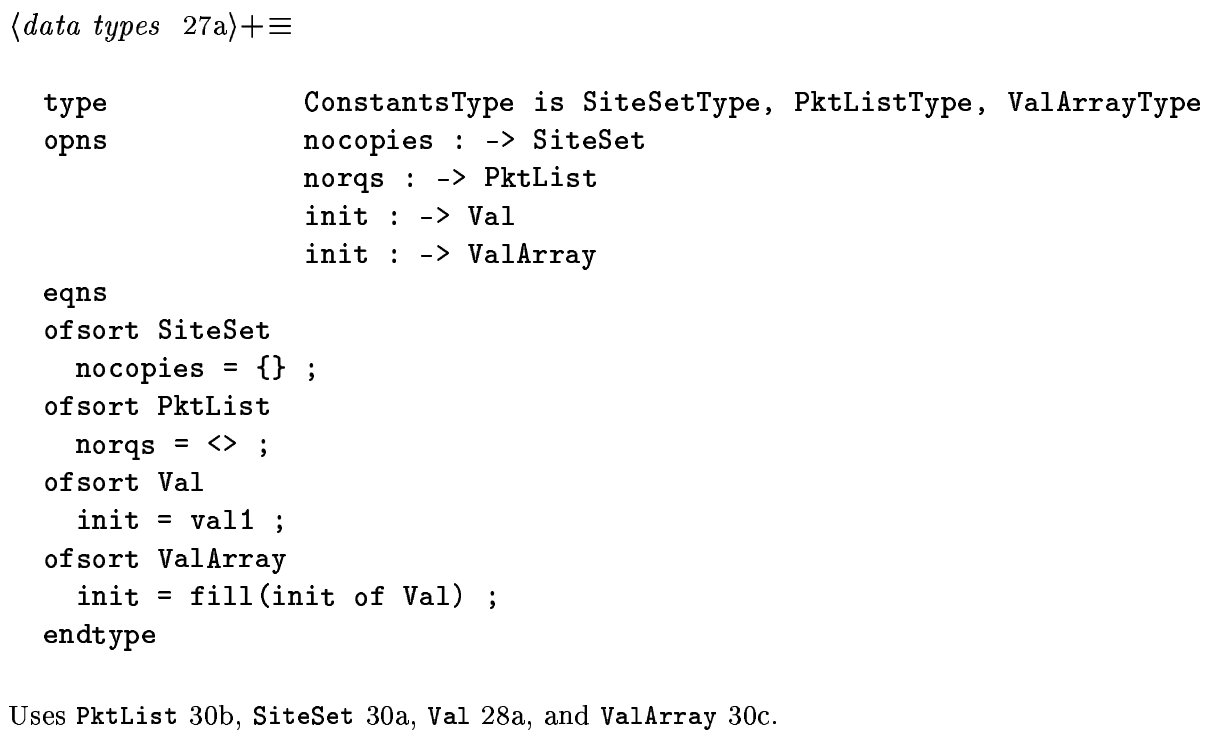

Uses PktList 30b, SiteSet 30a, Val 28a, and ValArray 30c.

\section{System Processes}

\section{D.1 CFS entity}

The process Site describes the management of a single block by a CFS site. This is a stateoriented specification, originally based on the state machine presented in [Fas96]. All state is specified as process parameters:

- state: State encodes the control part of the state.

- copies:SiteSet holds the set of other sites that have obtained a copy of the block from this master site.

- rqs : PktList holds the list of pending requests from other sites received while this site is writing.

As a special case, the first site to request (read or write) access to the block receives initial mastership. This is modelled as a firstmaster message received before the readrq or writerq has been sent. 
Note: for simplification, initial mastership assignment is not covered in the generated models. Instead, mastership is given arbitrarily to site1.

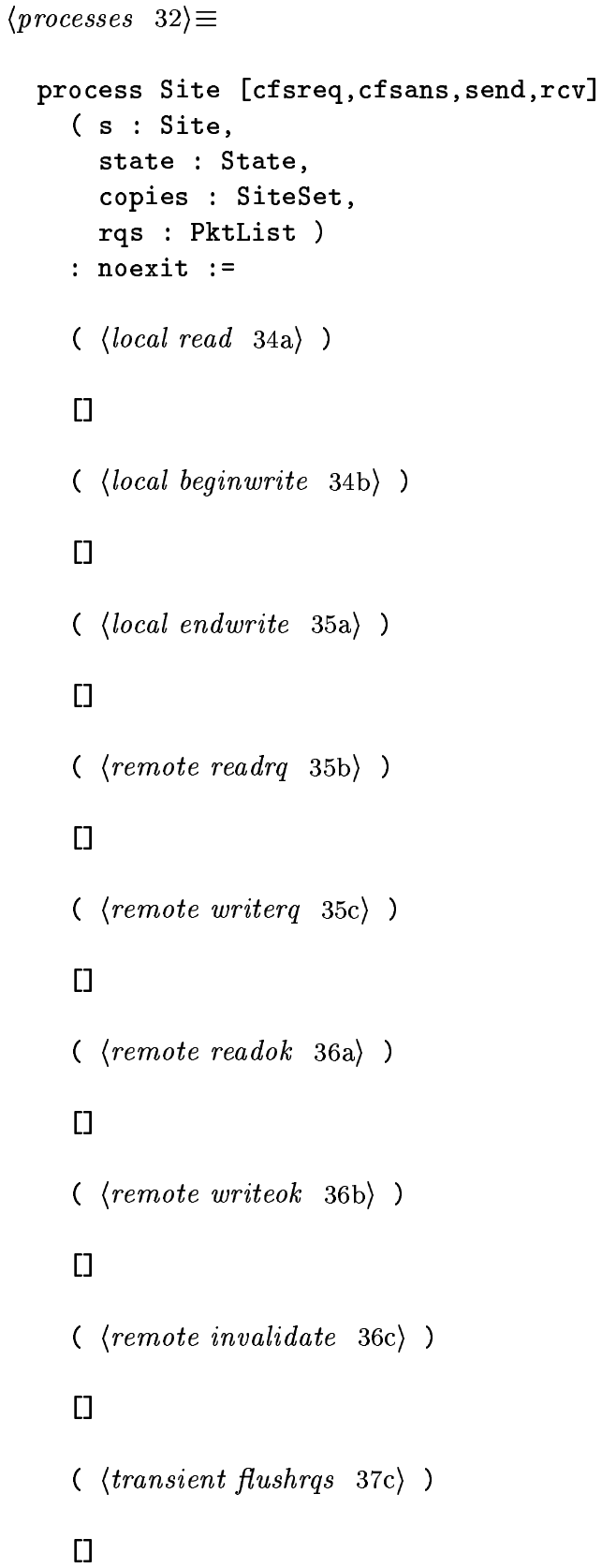




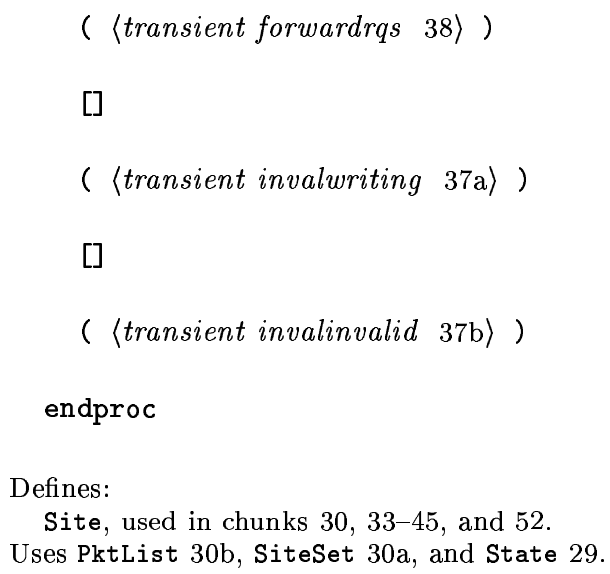

InitSite defines a site in initial state, i.e. with no valid copy of the block and no mastership.

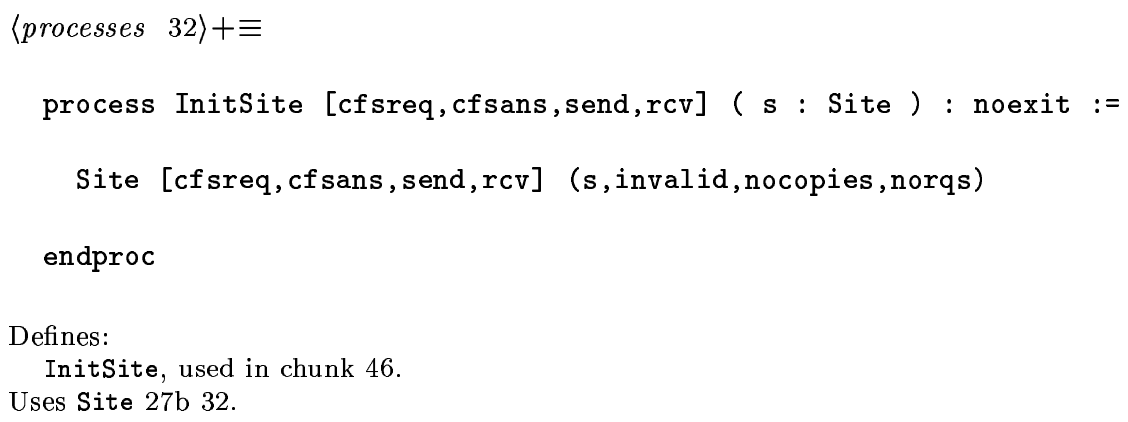

\section{D.1.1 Local Requests}

The following paragraphs detail the handling of CFS requests from local applications. 


\section{local read}

$34 \mathrm{a}$

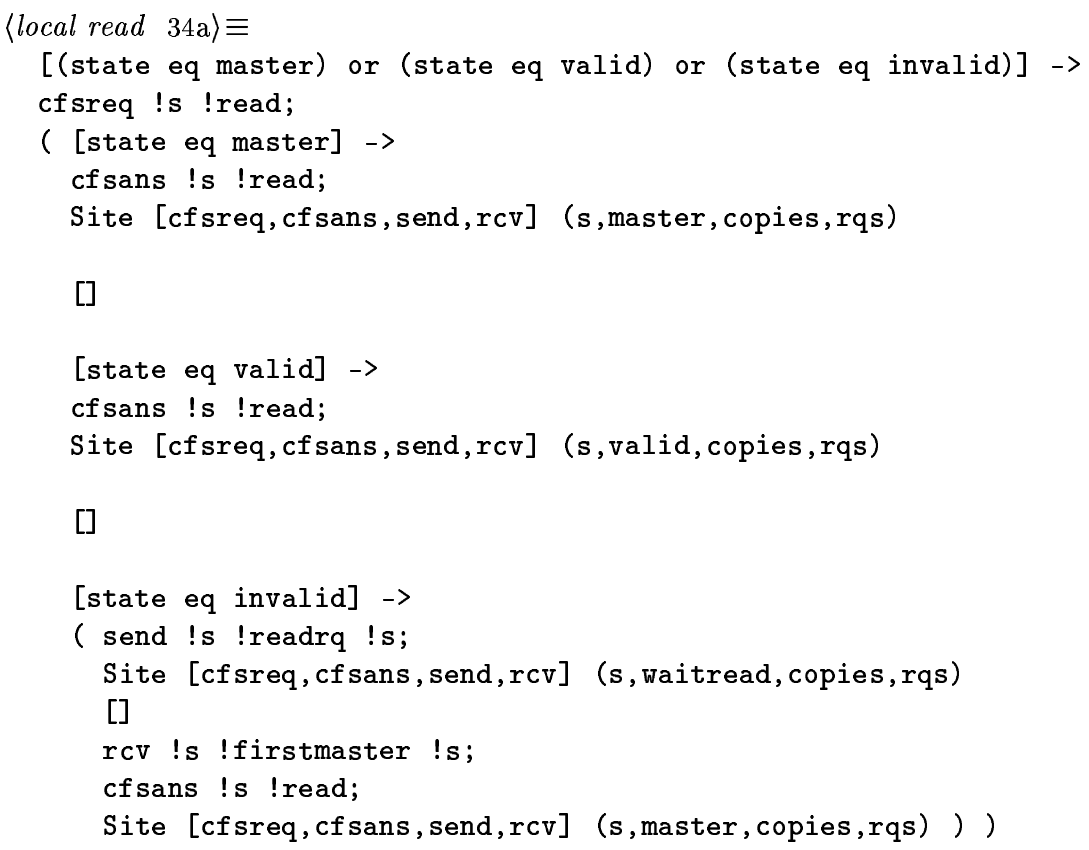

\section{local beginwrite}

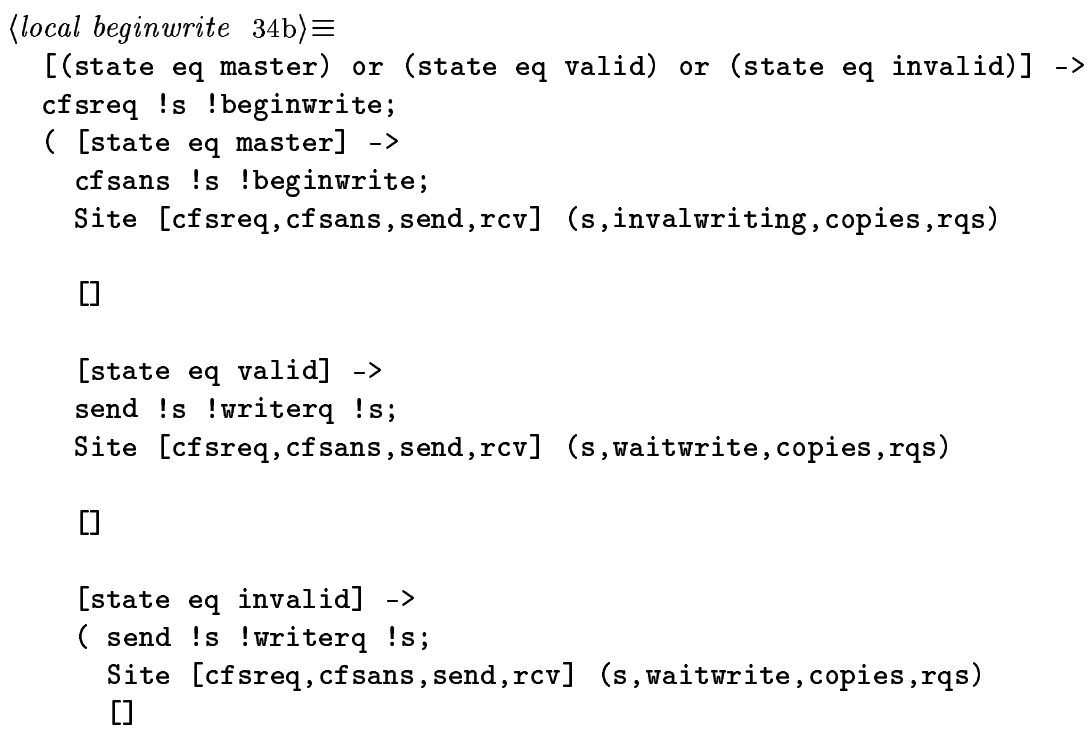




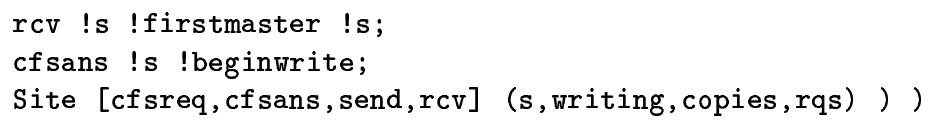

local endwrite

35a $\langle$ local endwrite $35 \mathrm{a}\rangle \equiv$

[state eq writing] ->

cfsreq !s !endwrite;

cfsans !s !endwrite;

Site [cfsreq, cf sans, send,rcv] (s,flushrqs, copies,rqs)

Uses Site 27b 32.

\section{D.1.2 Remote Messages}

The following paragraphs detail the handling of CFS protocol messages received from remote CFs sites.

\section{remote readrq}

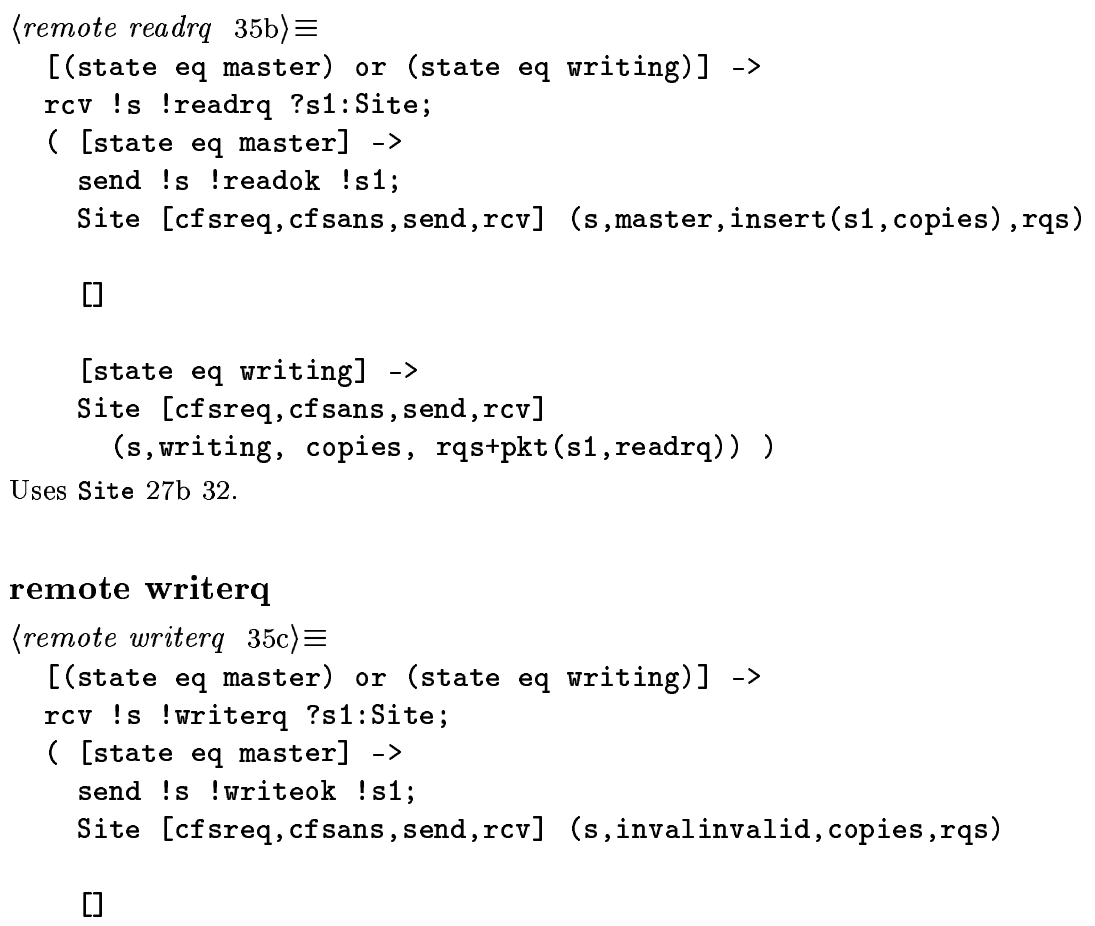




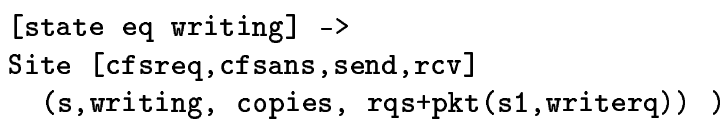

remote invalidate Note: unexpected reception of invalidate is possible in any state other than valid. This has been observed as a cause of deadlock in previous versions of this specification. These cases have been added in the specification; the message is ignored in these cases.

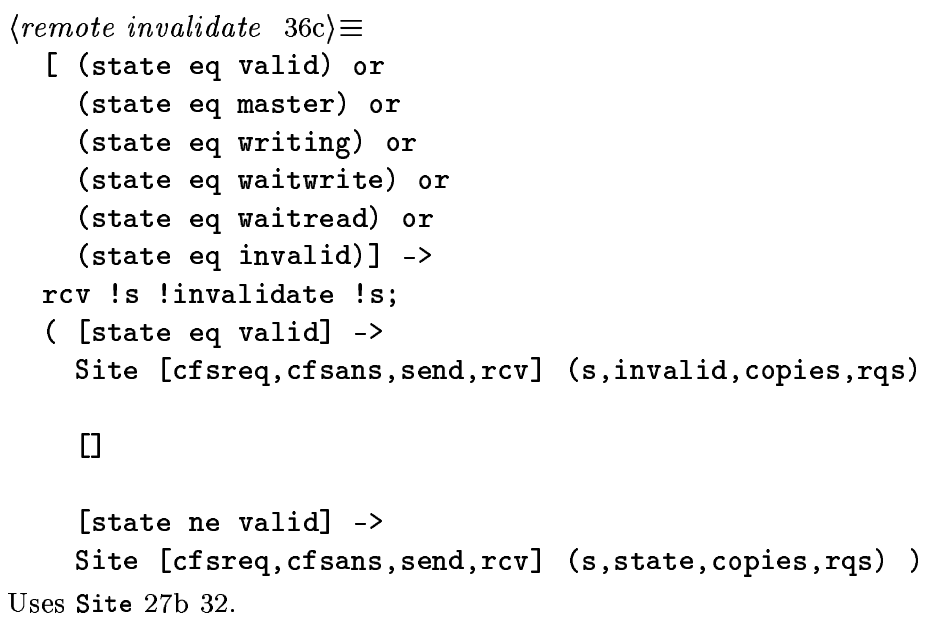




\section{D.1.3 Transient States}

The following paragraphs detail the processing done in transient states. Typically this involves flushing some internal list and sending corresponding messages.

transient invalwriting Invalidate remote copies in copies before going to writing.

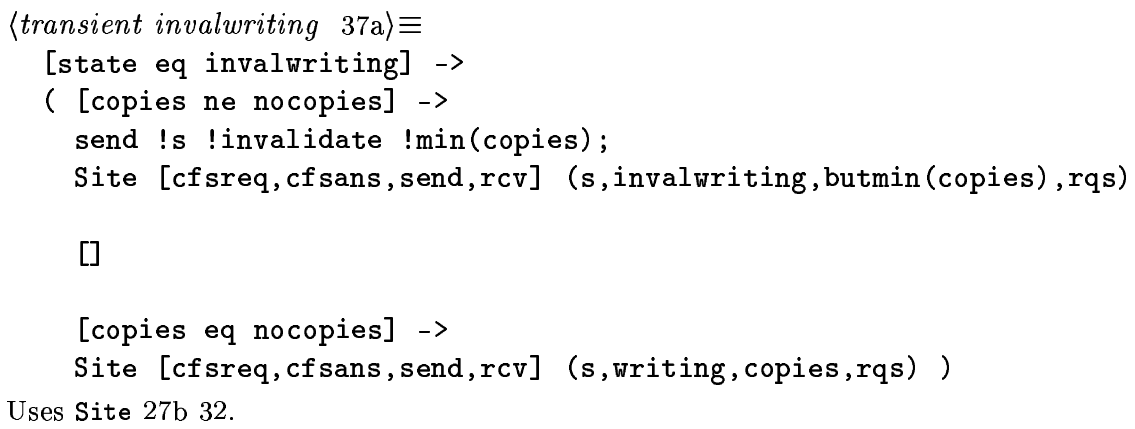

transient invalinvalid Invalidate remote copies in copies before going to invalid.

$37 \mathrm{~b}$

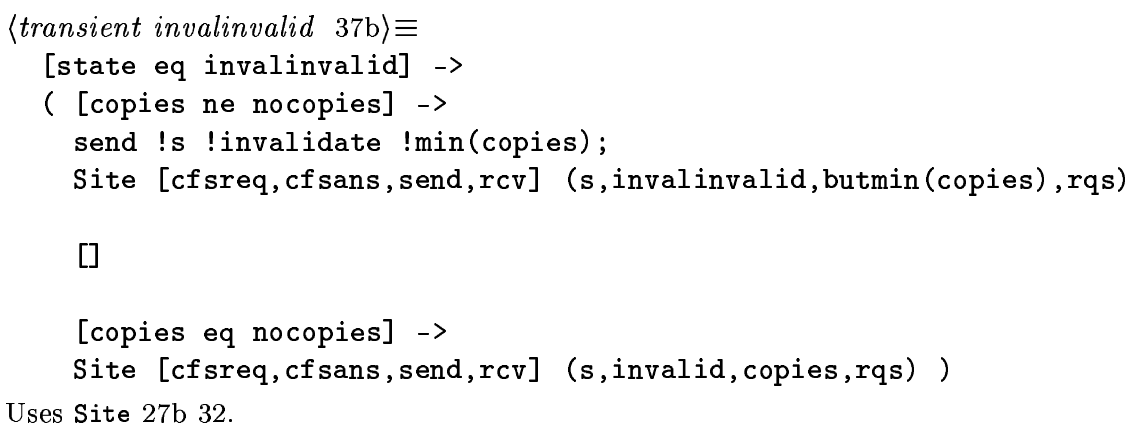

transient flushrqs Answer the pending requests in rqs.

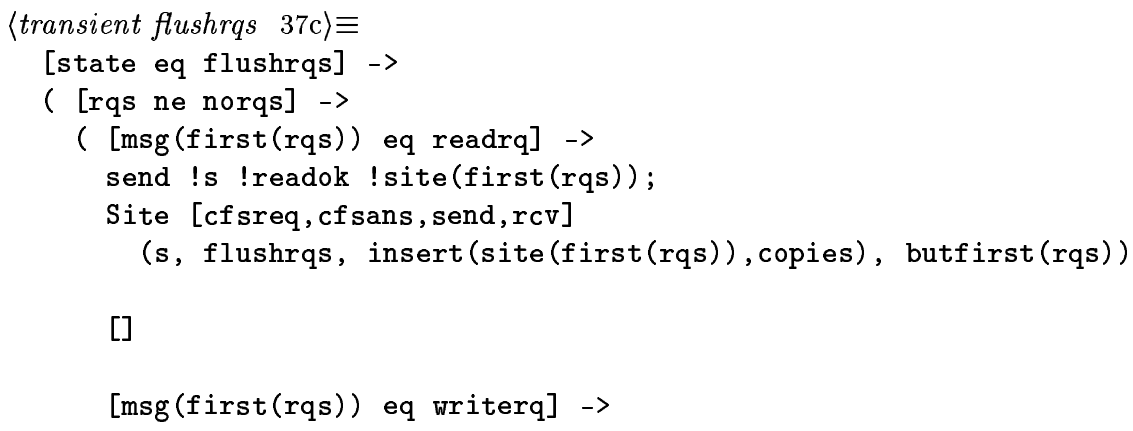


send !s !writeok !site(first(rqs));

Site [cfsreq, cfsans, send,rcv] (s,forwardrqs, copies, butfirst(rqs)) )

[]

[rqs eq norqs] ->

Site [cfsreq, cfsans, send,rcv] (s,master, copies,rqs) )

Uses Site 27b 32.

transient forwardrqs Invalidate remote copies in copies, then forward pending requests in rqs to the current master.

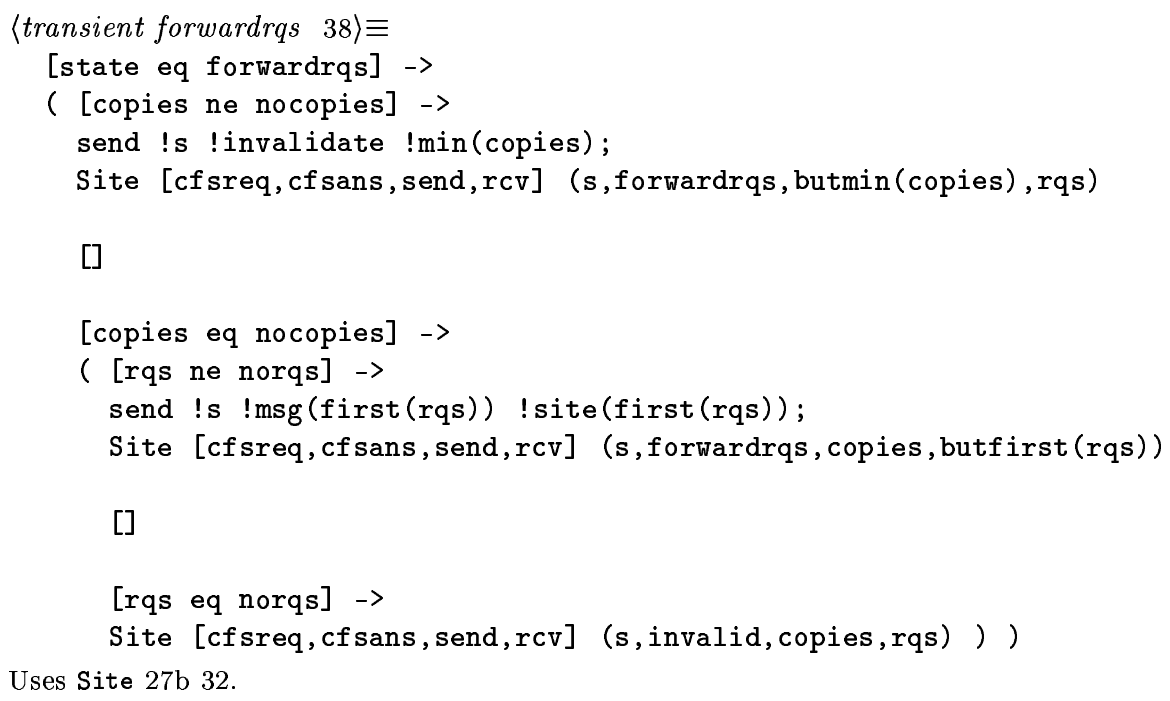

\section{D.2 Communication Channel}

The following processes define the medium through which CFS sites communicate. All events on send and rcv have the following attributes:

send ?s1 : Site ?m : Msg ?s2 : Site

rcv ?s1 : Site ?m : Msg ?s2 : Site

$\mathrm{s} 1$ is the site that sends/receives the message; $\mathbf{s} 2$ is the site concerned by the message. The channel ignores $\mathbf{s} 1$ and keeps s2. Note that no destination address is given; each site is responsible for accepting only the messages it is supposed to receive. This works because each kind of message has a well-defined destination: requests go to the master, responses go to the concerned site.

OutputCell is a one-slot bounded buffer whose input is restricted to a single site. The restriction to a single message avoids state space explosion. Using a different channel for 
each site allows messages from different sites to be received in any order (and blows up the state space). This is necessary for a correct working of the protocol; deadlocks have been observed in models with a single common channel.

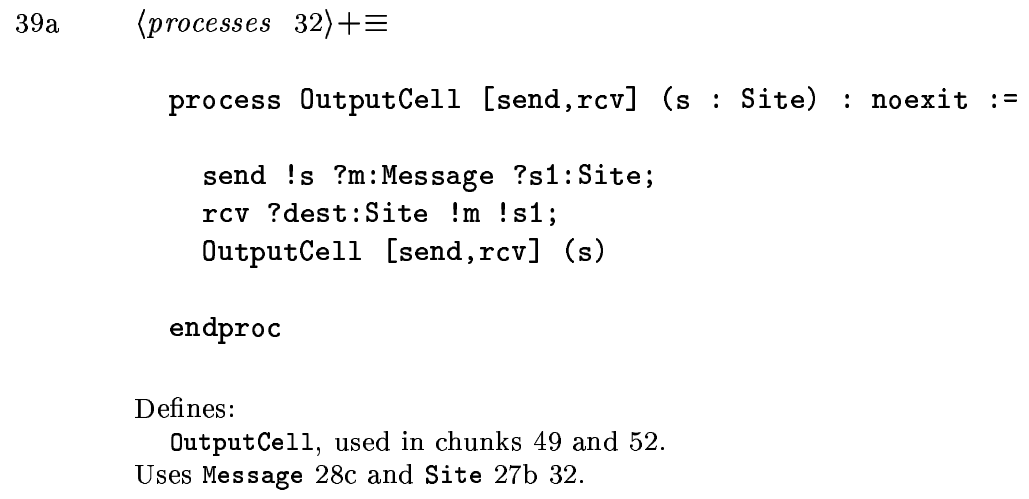

\section{D.3 Memory}

Memory holds the data (of sort Val) of the block controlled through the CFS protocol. Different copies are kept for each site. The CFs messages are seen through gate ctrl and cause data to be transfered on readok and writeok messages. Gates read and write model the access to memory by the application, with the following profiles:

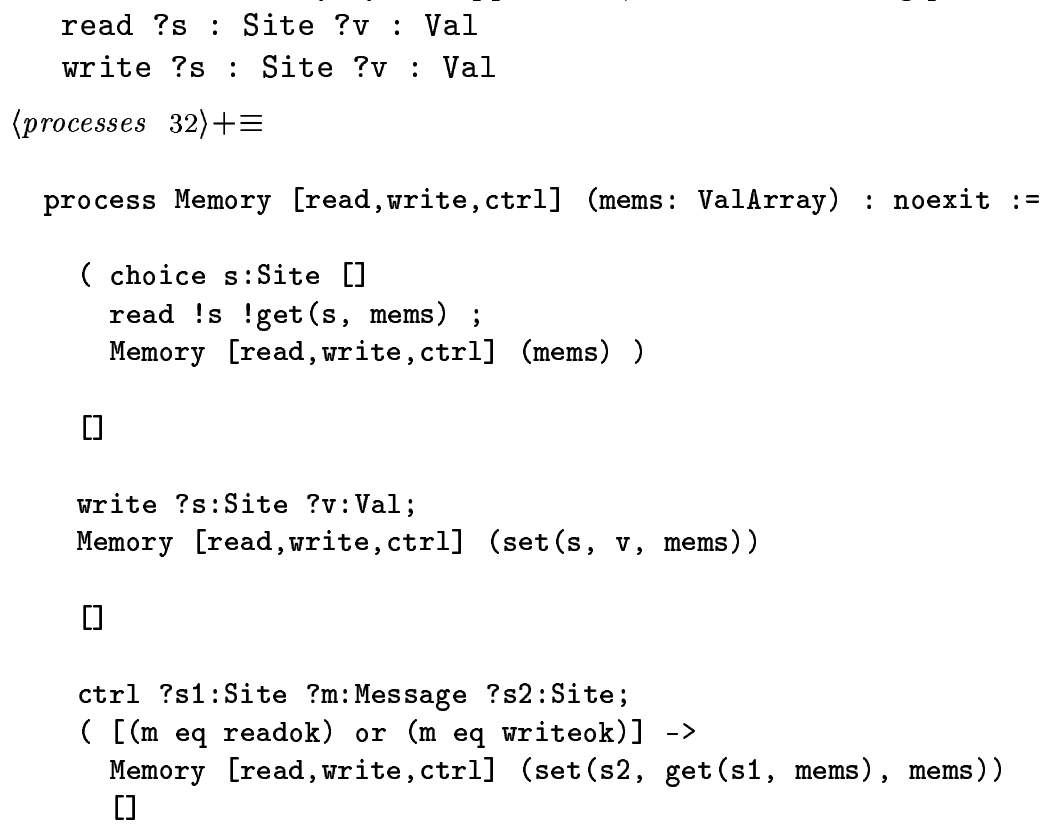


[(m ne readok) and ( $m$ ne writeok) $]->$

Memory [read,write, ctrl] (mems))

endproc

process InitMemory [read,write, send] : noexit :=

Memory [read,write, send] (init of ValArray)

endproc

Defines:

InitMemory, used in chunk 52 .

Memory, never used.

Uses Message 28c, Site 27b 32, Val 28a, and ValArray 30c.

\section{E Environment processes}

This section defines processes which describe the expected behaviour of the environment of a CFS system. These processes are used to filter out impossible execution paths when generating those components separately, in a compositional approach.

\section{E.1 Environment for Sites}

MasterSiteProxy, SlaveSiteProxy abstract the behaviour of another site, as seen from a given site through gates send and rcv. MasterSiteProxy covers messages to and form a master site, independently of its number; SlaveSiteProxy covers messages to and from a given slave site.

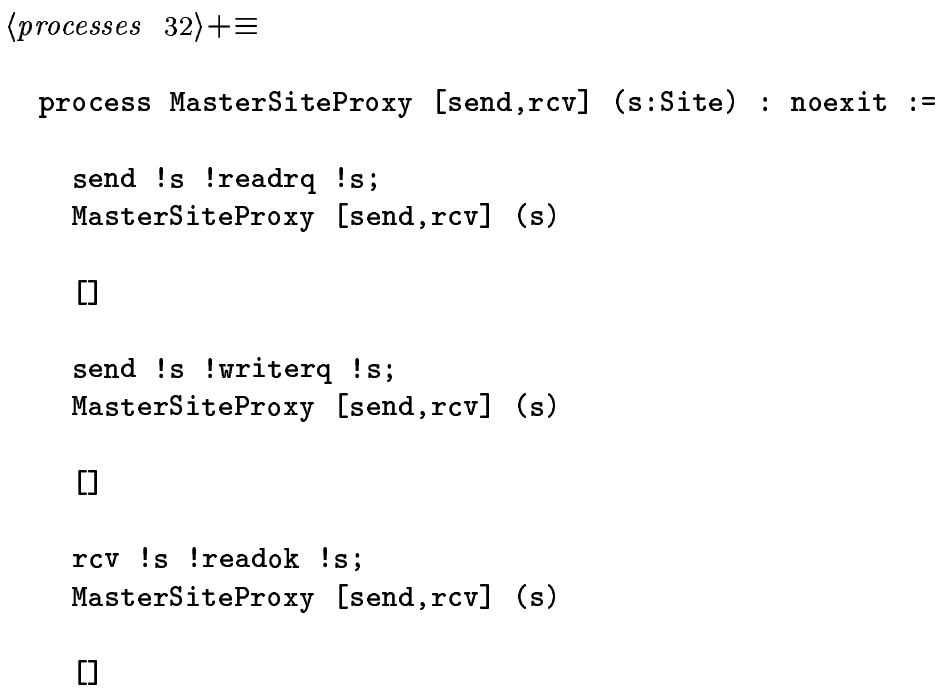




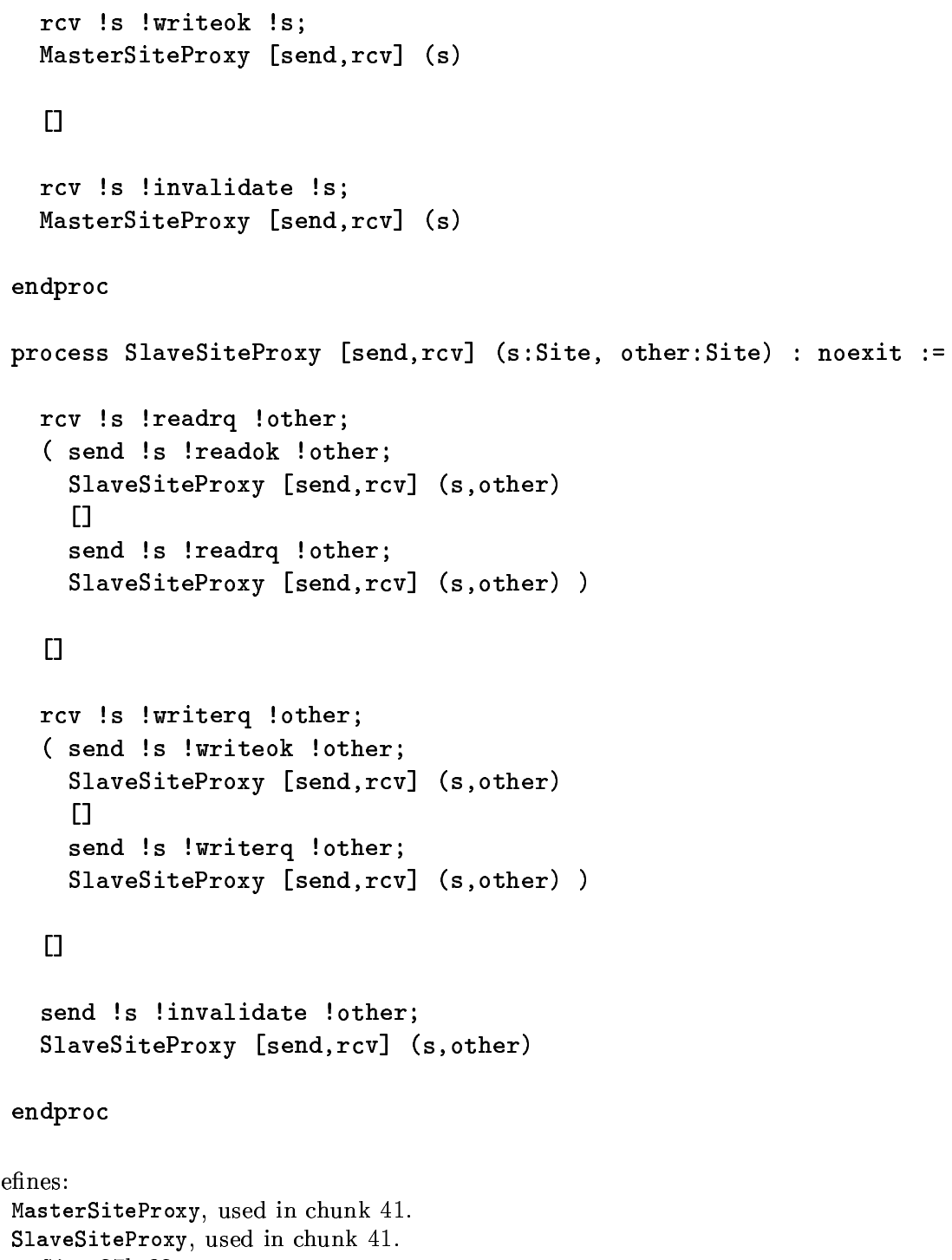

To constitute an environment for a given site, Site2Proxy and Site3Proxy combine a single MasterSiteProxy with one SlaveSiteProxy for one and two other sites, respectively. It is not necessary to include a SlaveSiteProxy for the constrained site, because in no case can a site become its own master: it cannot receive a readrq or writerq from itself, nor need to send an invalidate to itself. 


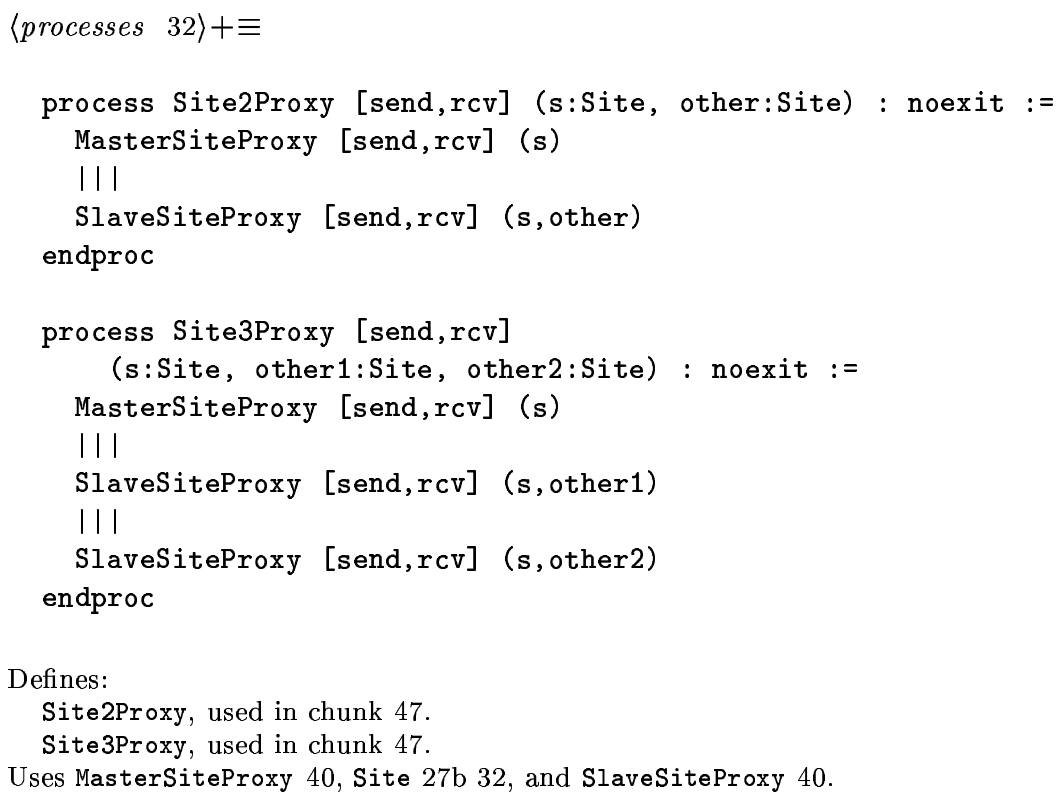

\section{E.2 Environment for Channels}

SlaveSendProxy, MasterSendProxy fix the messages sent by a site on its output channel, resp. in slave and master state. Note that the former depends only on the sender while the latter also depends on the receiver. They are used for restricting the environment of channel processes.

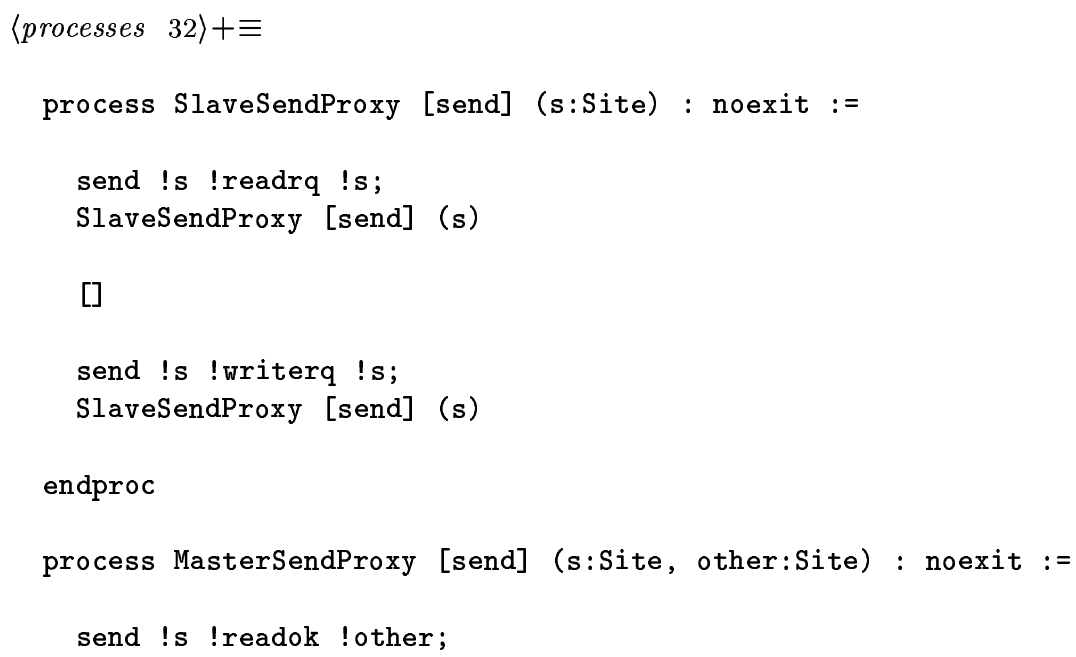




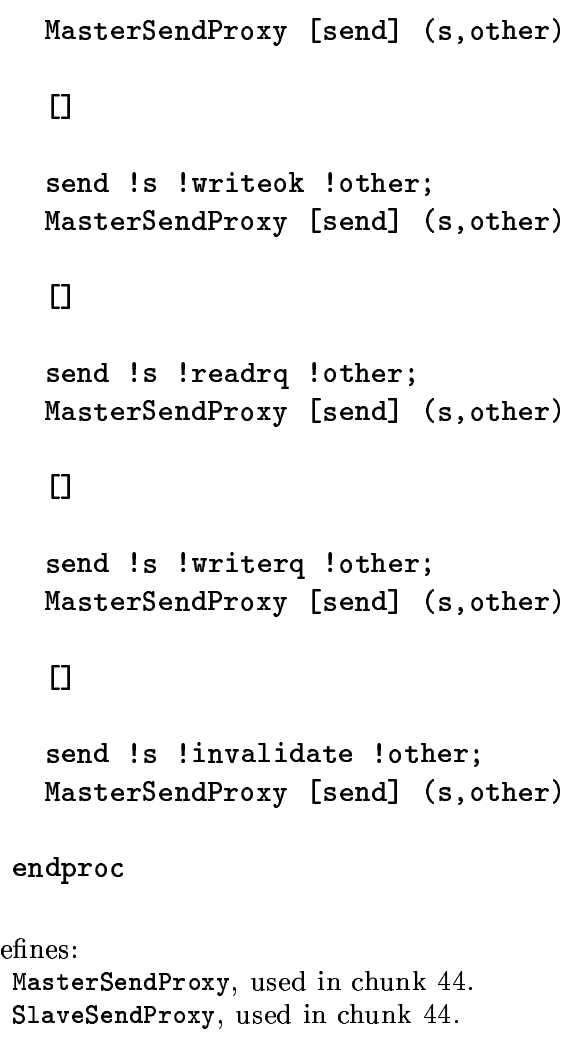

RcvProxy fixes message received from some channel by another site. It is used for restricting the environment of channel processes.

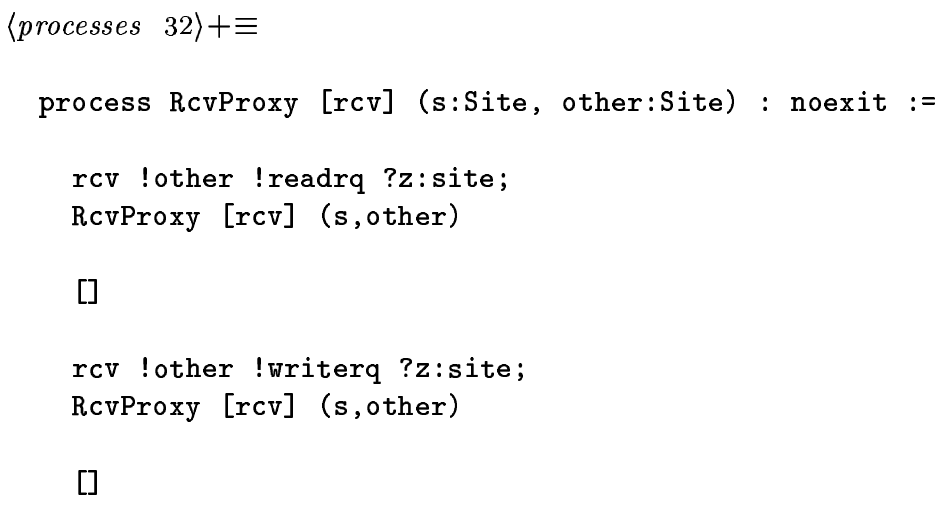


rcv !other !readok !other;

RcvProxy [rcv] (s,other)

[]

rcv !other !writeok !other;

RcvProxy [rcv] (s,other)

[]

rcv !other !readrq !other;

RcvProxy [rcv] (s, other)

[]

rcv !other !writerq !other;

RcvProxy [rcv] (s,other)

[]

rcv !other !invalidate !other;

RcvProxy [rcv] (s, other)

endproc

Defines:

RcvProxy, used in chunk 44.

Uses Site 27b 32.

Channel2Proxy and Channel3Proxy combine channel proxies to constrain a given channel, according to the expected number of sites (two and three, respectively). With the same reasoning as for site proxies, we can safely omit communications from a site to itself.

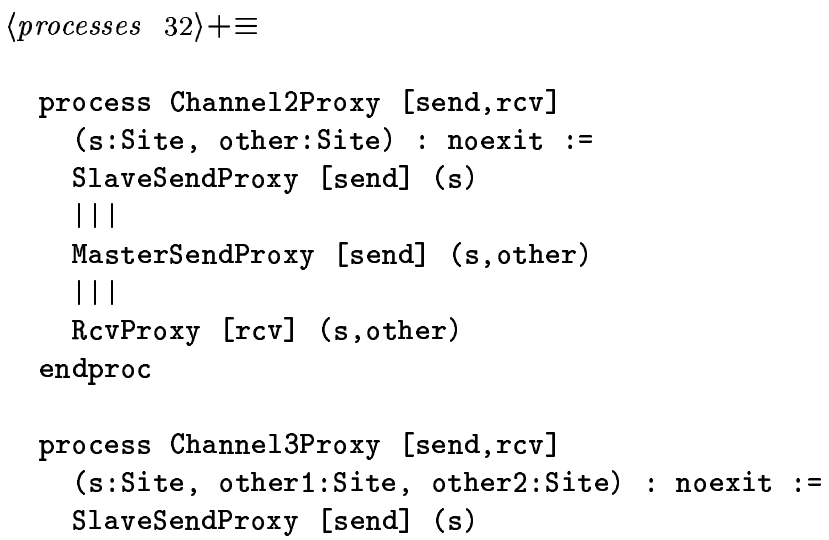


III

MasterSendProxy [send] (s,other1)

III

MasterSendProxy [send] (s,other2)

III

RcvProxy [rcv] (s,other1)

III

RcvProxy [rcv] (s, other2)

endproc

Defines:

Channel2Proxy, used in chunk 50.

Channel3Proxy, used in chunk 50.

Uses MasterSendProxy 42, RcvProxy 43, Site 27b 32, and SlaveSendProxy 42.

\section{E.3 User behaviour}

Process GeneralUser links calls to CFS and accesses to memory. It encodes the expected use of CFS by the application:

- call (request/answer) read then read the block any number of times;

- call beginwrite and endwrite before and after writing and/or reading the block any number of times.

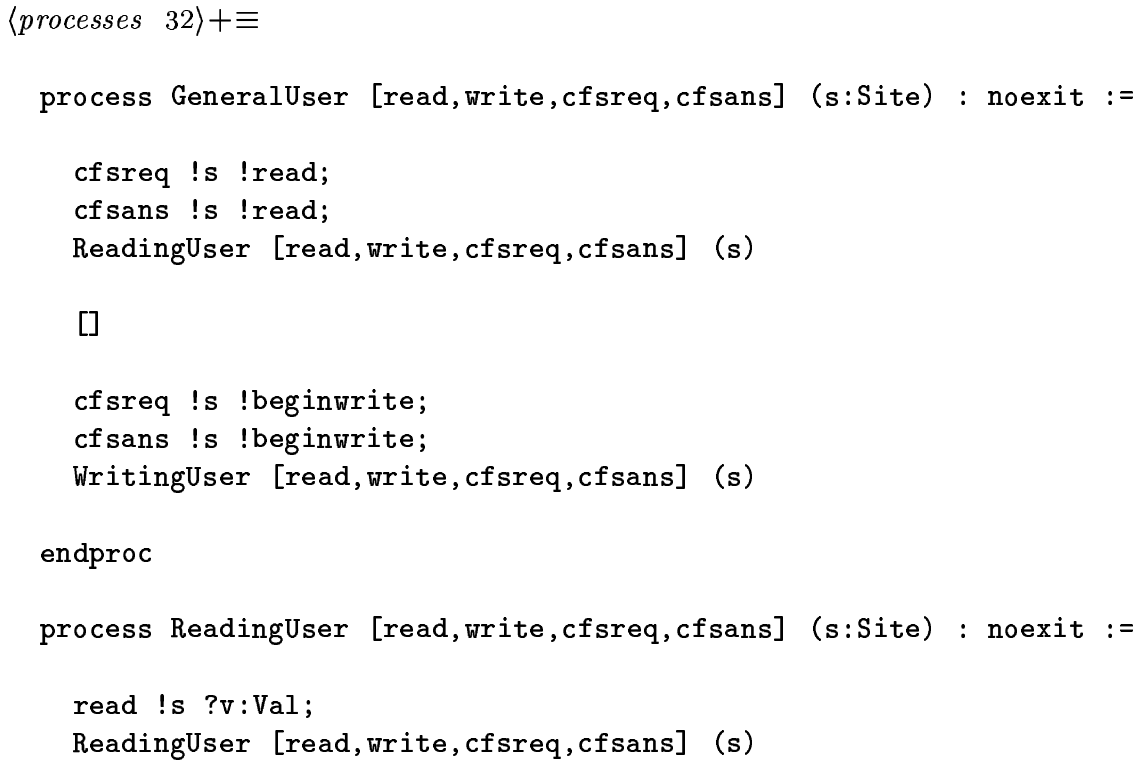




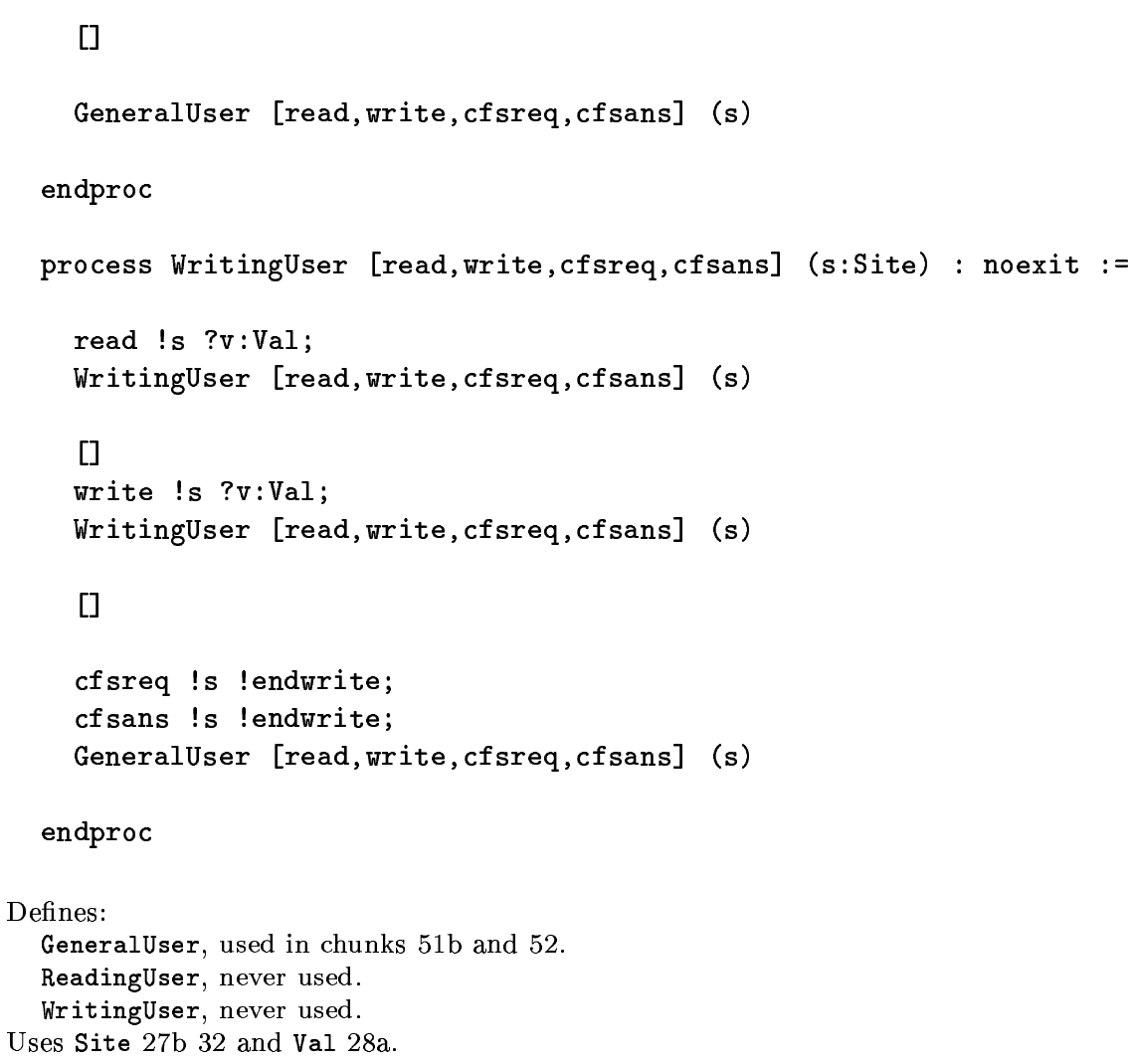

\section{F Instanciated Processes}

This section defines instances of previously defined processes as parameter-less processes. They are used with CAESAR's - root option to generate models of system components in a compositional approach.

\section{Site instances}

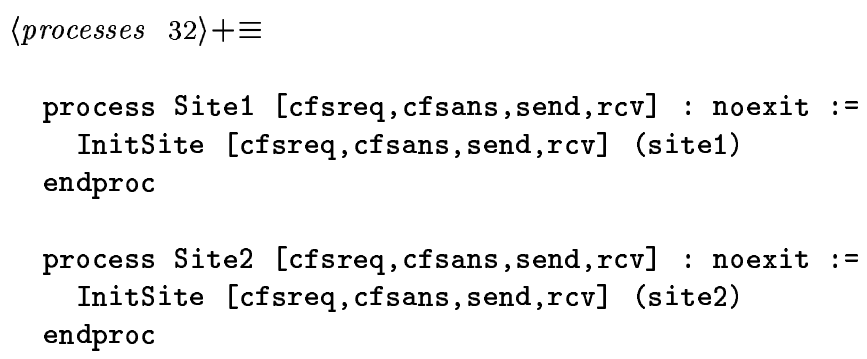




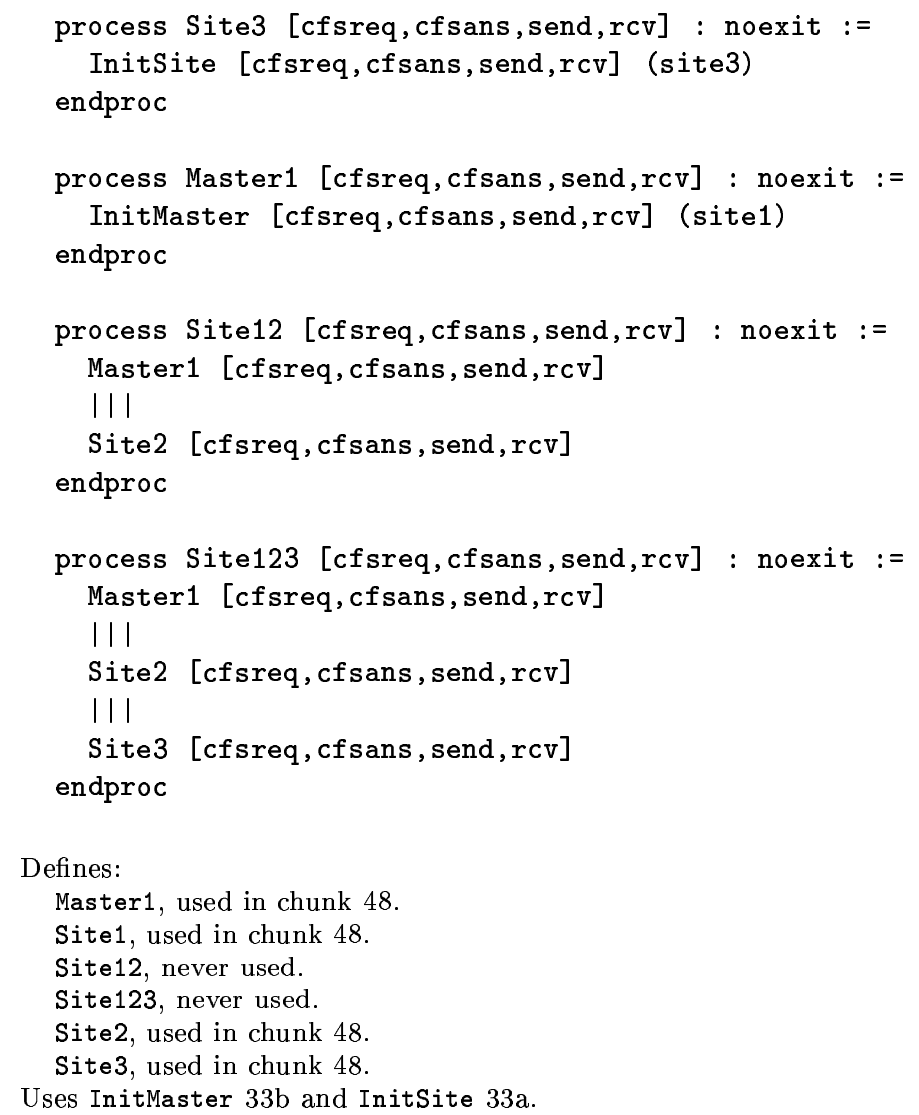

\section{Proxy instances}

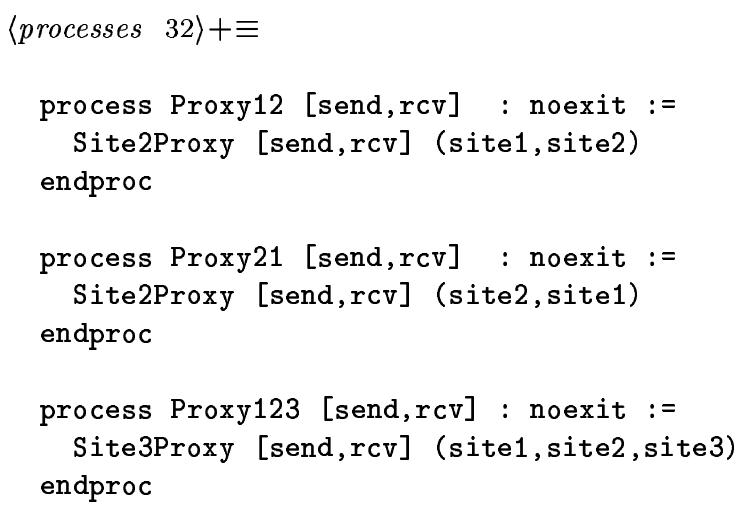




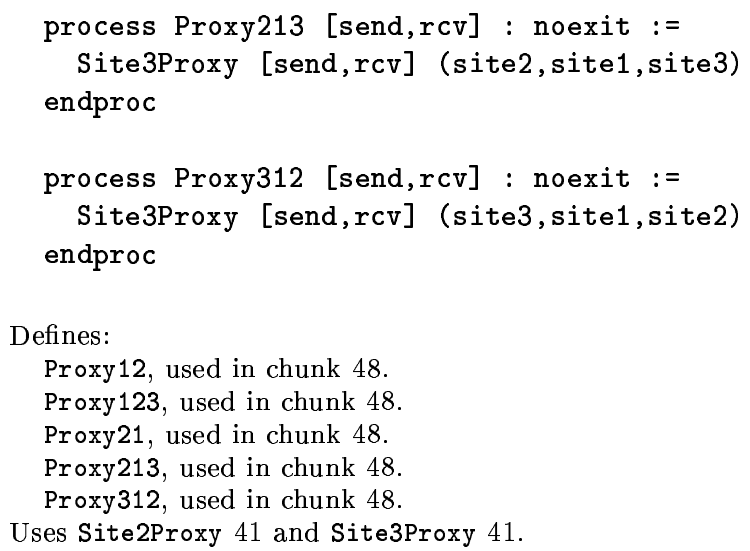

\section{Site instances with proxies}

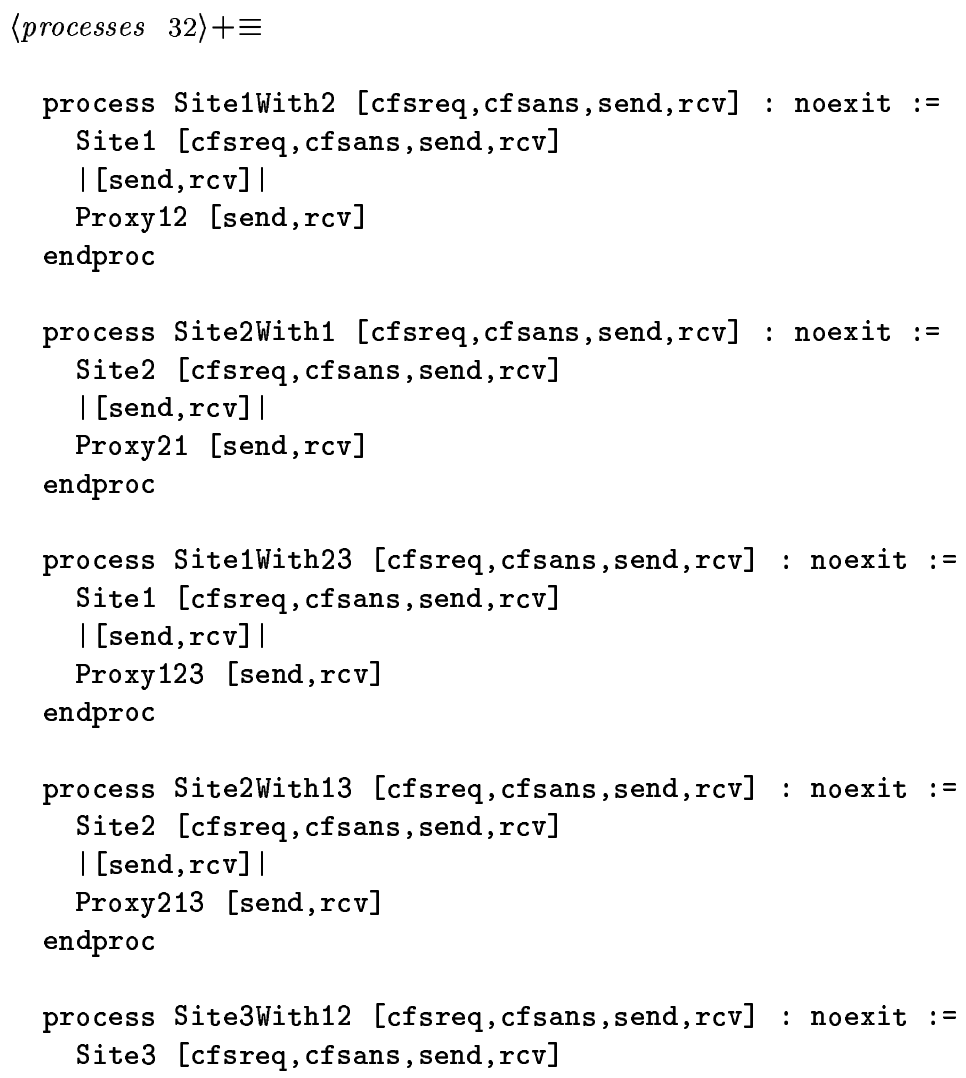




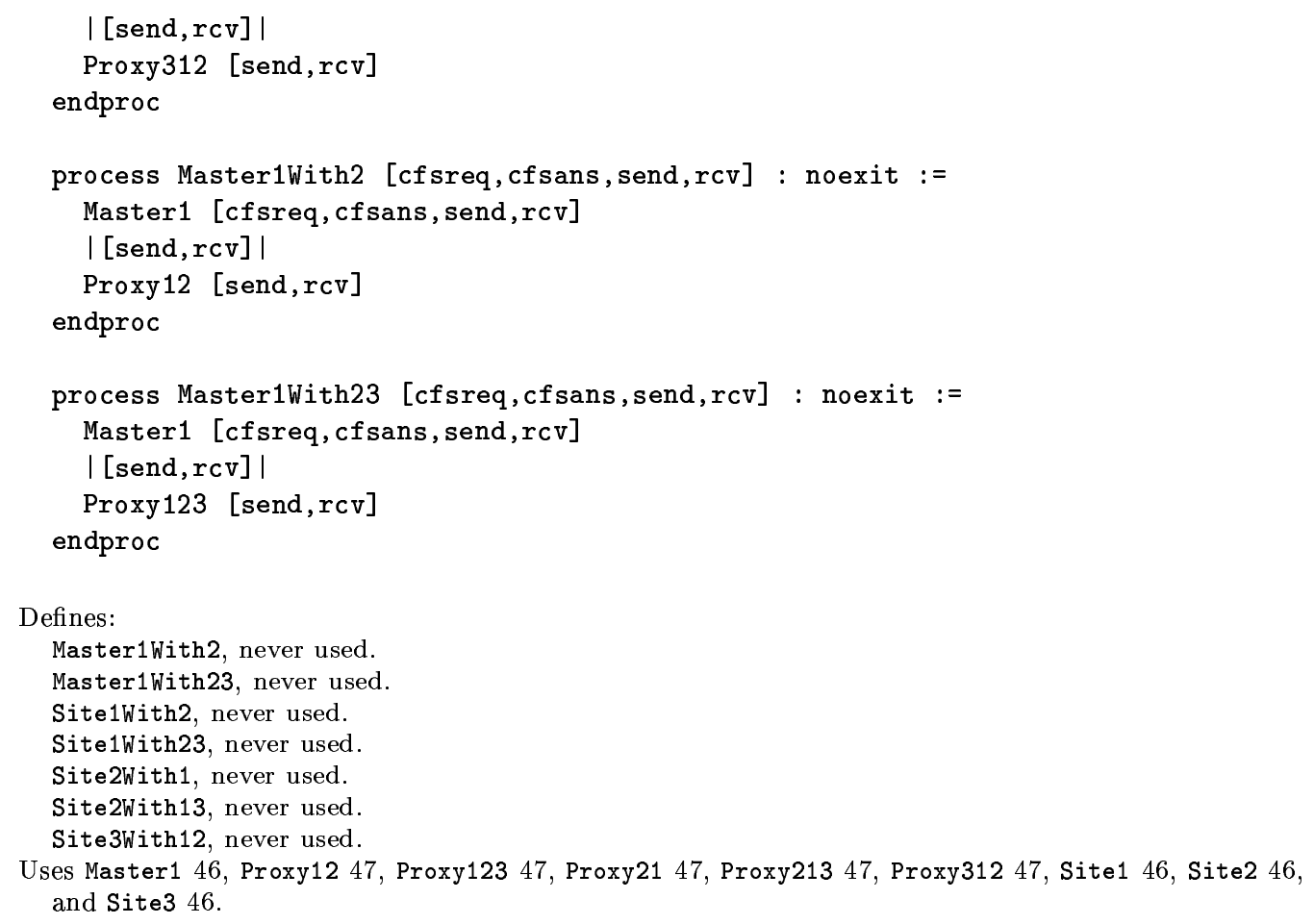

\section{Cell instances}

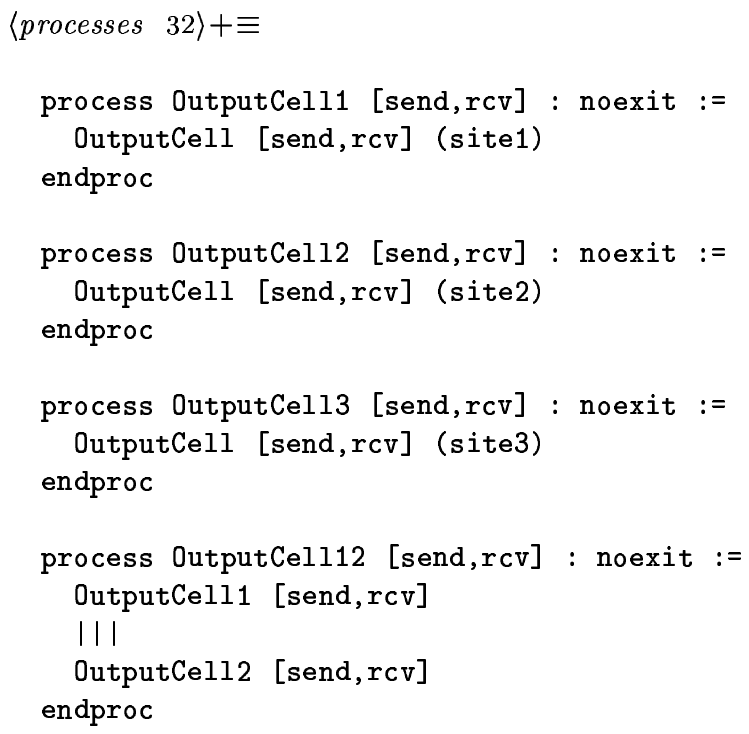




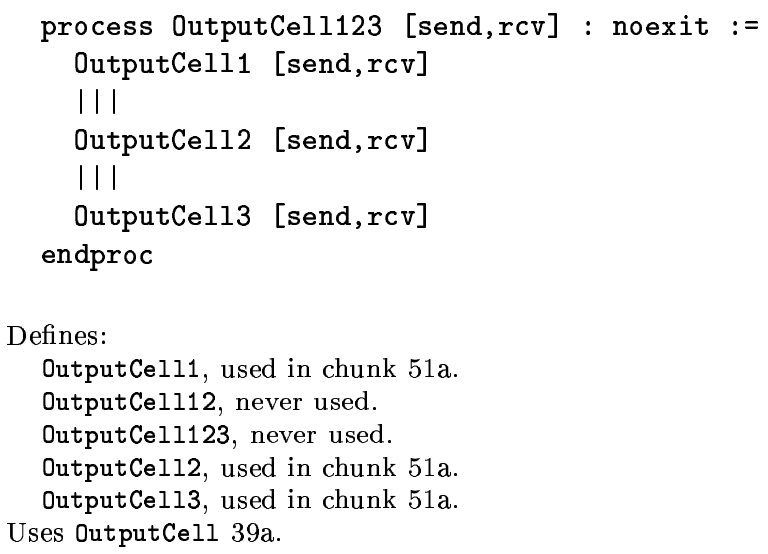

\section{Channel proxy instances}

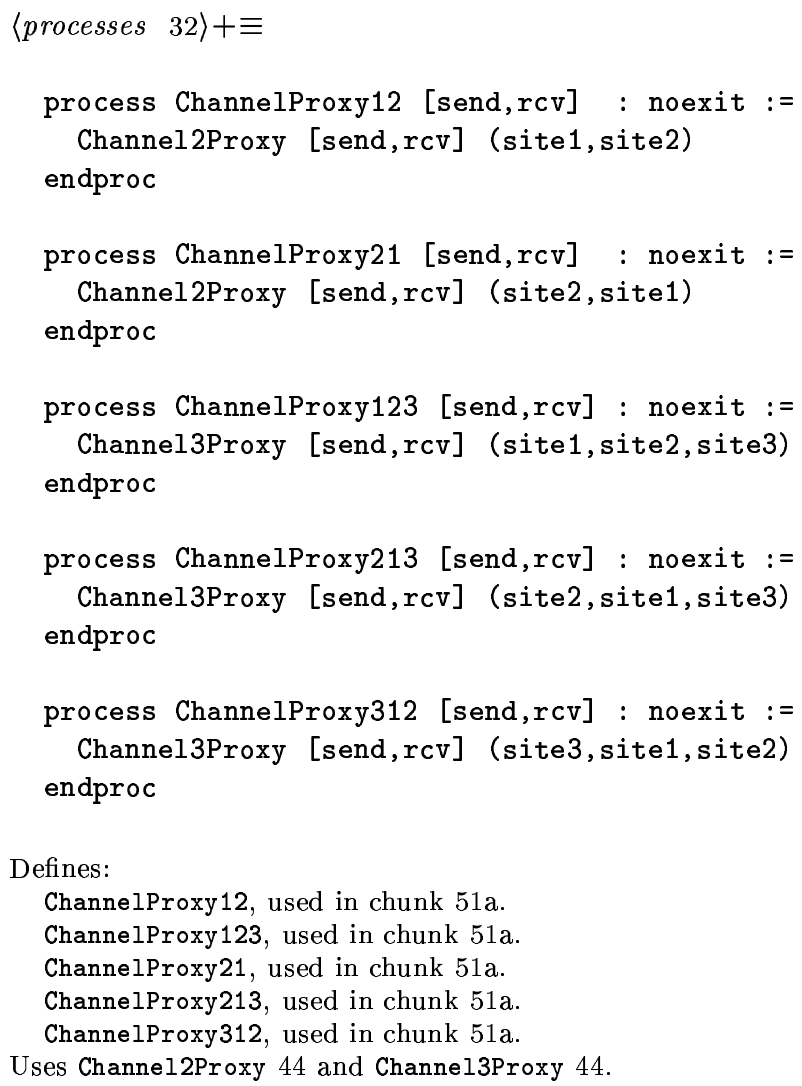




\section{Cell instances with proxies}

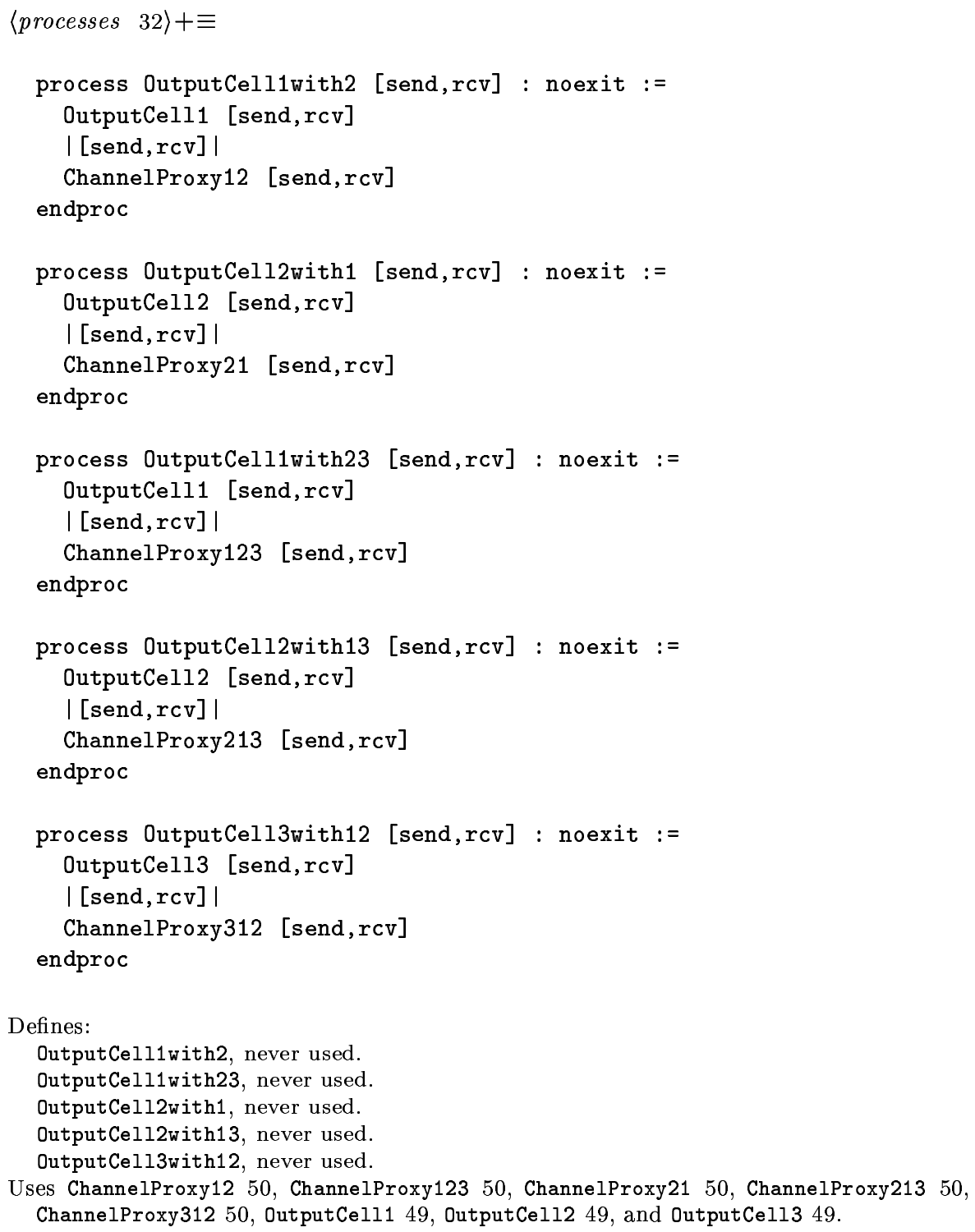

\section{General User instances}

$51 \mathrm{~b}$

$$
\begin{aligned}
& \langle\text { processes } 32\rangle+\equiv \\
& \text { process GeneralUser1 [read,write,cfsreq, cfsans] : noexit := }
\end{aligned}
$$




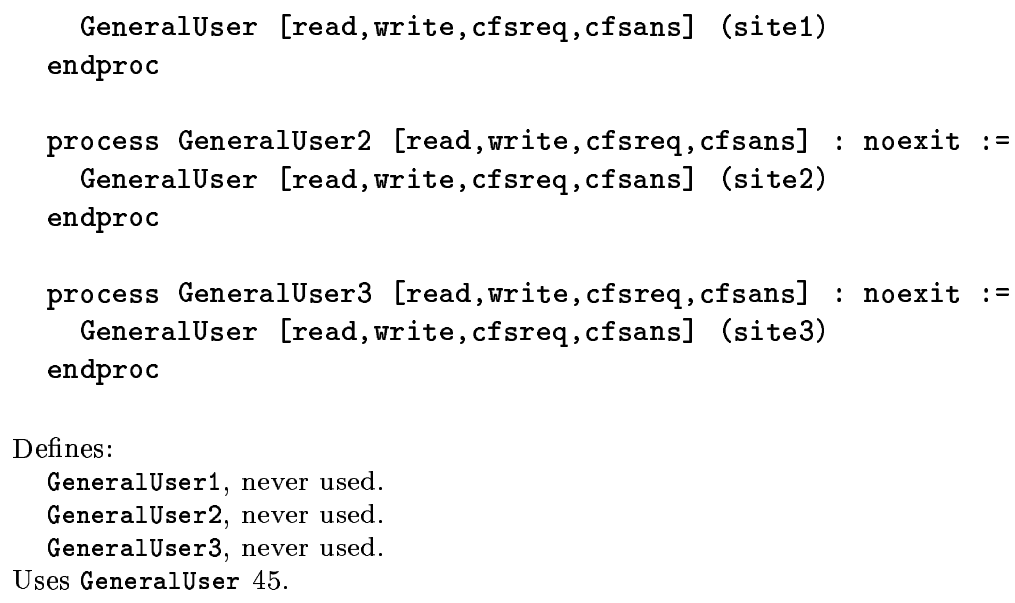

\section{G Top Level specification}

Note: the models used for the validation of CFs have been generated compositionally, using the instanciated processes above to produce separate components. The following top-level behaviour is given for illustration only; currently it cannot be compiled monolithically within available memory.

The specification covers the management of and access to a single block by three concurrent sites. An initial firstmaster message is generated spontaneously before the channel starts its normal operation.

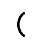




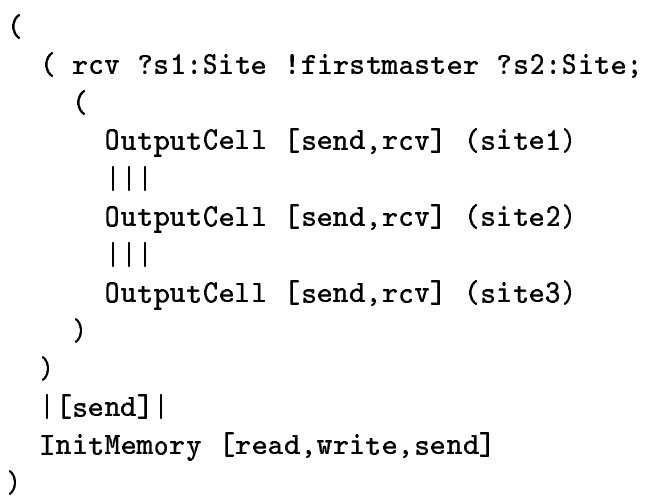

Finally, here is the specification itself.

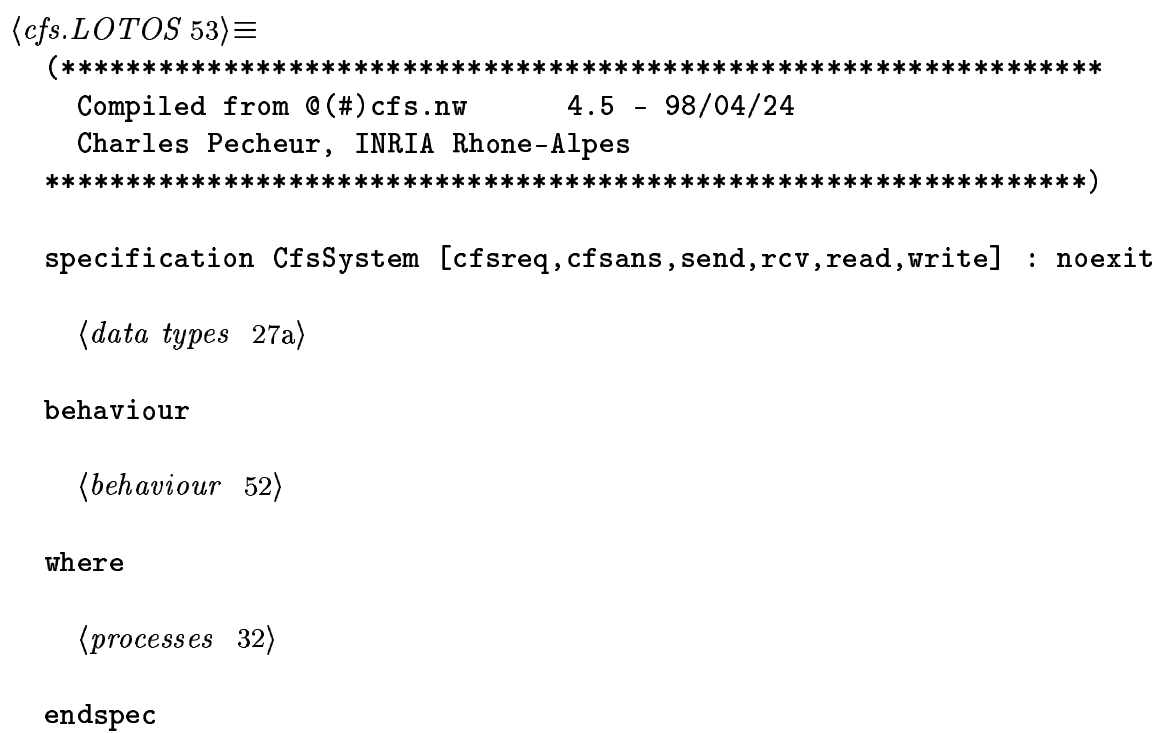

This code is written to file cfs.LOTOS. 


\title{
Index of LOTOS Identifiers
}

\author{
Bool: $27 \mathrm{a}, 29$ \\ CfsCall: $\underline{28 \mathrm{~b}}$ \\ Channel2Proxy: $\underline{44}, 50$ \\ Channel3Proxy: $\underline{44}, 50$ \\ ChannelProxy12: $\underline{50}, 51 \mathrm{a}$ \\ ChannelProxy123: $\underline{50}, 51 \mathrm{a}$ \\ ChannelProxy21: $\underline{50}, 51 \mathrm{a}$ \\ ChannelProxy213: $\underline{50}, 51 \mathrm{a}$ \\ ChannelProxy312: $\underline{50}, 51 \mathrm{a}$ \\ GeneralUser: $\underline{45}, 51 \mathrm{~b}, 52$ \\ GeneralUser 1: $51 \mathrm{~b}$ \\ GeneralUser2: $\underline{51 \mathrm{~b}}$ \\ GeneralUser3: $\underline{51 b}$ \\ InitMaster: $\underline{33 \mathrm{~b}}, 46$ \\ InitMemory: $\underline{39 \mathrm{~b}}, 52$ \\ InitSite: $\underline{33 \mathrm{a}}, 46$ \\ Master1: $\underline{46}, 48$ \\ MasterSendProxy: $\underline{42}, 44$ \\ MasterSiteProxy: $\underline{40}, 41$ \\ Master1With2: $\underline{48}$ \\ Master1With23: $\underline{48}$ \\ Memory: $39 \mathrm{~b}$ \\ Message: $\underline{28 \mathrm{c}}, 30 \mathrm{~b}, 39 \mathrm{a}, 39 \mathrm{~b}$ \\ OutputCell: $39 \mathrm{a}, 49,52$ \\ OutputCell1: $\underline{49}, 51 \mathrm{a}$ \\ OutputCell12: $\underline{49}$ \\ OutputCell123: $\underline{49}$ \\ OutputCel12: $\underline{49}, 51 \mathrm{a}$ \\ OutputCel13: $\underline{49}, 51 \mathrm{a}$ \\ OutputCell1with2: \\ OutputCell1with23: $\underline{51 \mathrm{a}}$ \\ OutputCel12with1: $\underline{51 \mathrm{a}}$ \\ OutputCell2with13: $\underline{51 \mathrm{a}}$ \\ OutputCell3with12: $\underline{51 \mathrm{a}}$ \\ Pkt: $\underline{30 \mathrm{~b}}$ \\ PktList: $\underline{30 \mathrm{~b}}, 31,32$ \\ Proxy12: 47,48 \\ Proxy123: $\underline{47}, 48$ \\ Proxy21: 47, 48 \\ Proxy213: $\underline{47}, 48$ \\ Proxy312: $\underline{47}, 48$
}

INRIA 
RcvProxy: $\underline{43}, 44$

ReadingUser: $\underline{45}$

Site: $\underline{27 b}, 30 \mathrm{a}, 30 \mathrm{~b}, 30 \mathrm{c}, \underline{32}, 33 \mathrm{a}, 33 \mathrm{~b}, 34 \mathrm{a}, 34 \mathrm{~b}, 35 \mathrm{a}, 35 \mathrm{~b}, 35 \mathrm{c}, 36 \mathrm{a}, 36 \mathrm{~b}, 36 \mathrm{c}, 37 \mathrm{a}, 37 \mathrm{~b}$, $37 \mathrm{c}, 38,39 \mathrm{a}, 39 \mathrm{~b}, 40,41,42,43,44,45,52$

Site1: $\underline{46}, 48$

Site12: $\underline{46}$

Site123: $\underline{46}$

Site2: $\underline{46}, 48$

Site3: $\underline{46}, 48$

Site2Proxy: $\underline{41}, 47$

Site3Proxy: $\underline{41}, 47$

SiteSet: $30 \mathrm{a}, 31,32$

Site1With2: $\underline{48}$

Site1With23: $\underline{48}$

Site2With1: $\underline{48}$

Site2With13: $\frac{48}{48}$

Site3With12: $\underline{48}$

SlaveSendProxy: $\underline{42}, 44$

SlaveSiteProxy: $\underline{40}, 41$

State: $\underline{29}, 32$

Val: $28 \mathrm{a}, 30 \mathrm{c}, 31,39 \mathrm{~b}, 45$

ValArray: $\underline{30 \mathrm{c}}, 31,39 \mathrm{~b}$

WritingUser: $\underline{45}$

RR n3416 


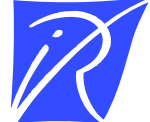

Unit`e de recherche INRIA Lorraine, Technopôle de Nancy-Brabois, Campus scientifique, 615 rue du Jardin Botanique, BP 101, 54600 VILLERS LÈS NANCY

Unit'e de recherche INRIA Rennes, Irisa, Campus universitaire de Beaulieu, 35042 RENNES Cedex

Unit'e de recherche INRIA Rhône-Alpes, 655, avenue de l'Europe, 38330 MONTBONNOT ST MARTIN

Unit'e de recherche INRIA Rocquencourt, Domaine de Voluceau, Rocquencourt, BP 105, 78153 LE CHESNAY Cedex

Unit'e de recherche INRIA Sophia-Antipolis, 2004 route des Lucioles, BP 93, 06902 SOPHIA-ANTIPOLIS Cedex

Éditeur

INRIA, Domaine de Voluceau, Rocquencourt, BP 105, 78153 LE CHESNAY Cedex (France) http://www.inria.fr

ISSN 0249-6399 